\title{
Implications of a light charged Higgs boson at the LHC run III in the 2HDM
}

\author{
Abdesslam Arhrib* \\ Faculté des Sciences et Techniques, Abdelmalek Essaadi University, B.P. 416, Tangier, Morocco
}

Rachid Benbrik ${ }^{\dagger}$ and Hicham Harouiz

Laboratoire de Physique Fondamentale et Applique Safi, Facult Polydisciplinaire de Safi, Safi, Morocco

Stefano Moretti

School of Physics and Astronomy, University of Southampton, Southampton, SO17 1BJ, United Kingdom

Yan Wang $\oplus^{\S}$

College of Physics and Electronic Information, Inner Mongolia Normal University, Hohhot 010022, PR China

Qi-Shu Yan"

School of Physics Sciences, University of Chinese Academy of Sciences, Beijing 100039, PR China and Center for future high energy physics, Chinese Academy of Sciences, Beijing 100039, PR China

(Received 10 April 2020; accepted 7 December 2020; published 30 December 2020)

\begin{abstract}
In this study, we focus on the bosonic decays of a light charged Higgs boson (i.e., with $M_{H^{ \pm}}<m_{t}-m_{b}$ ) in the 2-Higgs Doublet Model (2HDM) Type-I. To study the signal of such a charged Higgs state at the Large Hadron Collider (LHC), in a scenario where the $H^{0}$ boson is the Standard Model (SM)-like one already discovered, we assume that it decays mainly via $h^{0} W^{ \pm *}$ and/or $H^{ \pm} \rightarrow A^{0} W^{ \pm *}$ (i.e., via an off-shell $W^{ \pm}$boson), which can reach a sizable branching ratio (BR) for $\tan \beta \geq 4$, when the exclusion bounds from $H^{ \pm} \rightarrow \tau \nu$ and $c s$ searches get weaker. By using six benchmark points (BPs), which are consistent with current LHC constraints, we perform a Monte Carlo (MC) study and examine the sensitivity of the LHC to a light charged Higgs boson decaying via the above bosonic modes and produced in top decay following both single top and top pair production processes. Our findings demonstrate that, when the integrated luminosity can reach $300 \mathrm{fb}^{-1}$, the LHC has the potential to either discover or rule out most of these BPs via either of these two production and decay channels or both.
\end{abstract}

DOI: 10.1103/PhysRevD.102.115040

\section{INTRODUCTION}

Following the discovery of a $125 \mathrm{GeV}$ Higgs boson in the first run of the LHC [1,2], several studies of its properties have now been undertaken. These cover the measurement of its mass, width, spin, $C P$ properties, as well as couplings. The current situation is that the measured Higgs signal rates in all available channels agree with the

\footnotetext{
*aarhrib@gmail.com

r.benbrik@uca.ac.ma

s.moretti@soton.ac.uk

§wangyan@imnu.edu.cn

"yanqishu@ucas.ac.cn
}

Published by the American Physical Society under the terms of the Creative Commons Attribution 4.0 International license. Further distribution of this work must maintain attribution to the author(s) and the published article's title, journal citation, and DOI. Funded by SCOAP ${ }^{3}$.
SM predictions at the $\sim 2 \sigma$ level [3]. However, the possibility that the observed Higgs boson state (hereafter, referred to as $H_{\text {obs }}$ ) could belong to a model with an extended Higgs sector, such as the SM with an extra singlet, doublet, and/or triplet, has not been ruled out. Among such higher Higgs representations, those with an extra doublet or triplet also contain one or more charged Higgs bosons in their (pseudo)scalar spectrum. The discovery of such charged Higgs bosons would then be an eminent signal of a multiplet Higgs sector and thus, clear evidence of physics beyond the SM (BSM). However, nature has so far indicated that the Higgs mechanism relies upon a doublet structure, so we focus here on a BSM scenario that only employs this particular multiplet.

One of the simplest extensions of the SM within this kind is the 2HDM, which contains two Higgs doublets, $\Phi_{1}$ and $\Phi_{2}$, used to give mass to all fermions. The particle spectrum of the 2HDM is as follows: two $C P$-even $\left(h^{0}\right.$ and $H^{0}$, with $\left.M_{h^{0}}<M_{H^{0}}\right)$, one $C P$-odd $\left(A^{0}\right)$, and a pair of charged $\left(H^{ \pm}\right)$ 
Higgs bosons. At hadron colliders, a charged Higgs boson can be produced through several channels. Light charged Higgs states, i.e., with $M_{H^{0}}^{ \pm} \leq m_{t}-m_{b}$, are copiously induced by $t \bar{t}$ production followed by the top decay $t \rightarrow b H^{+}$(or the equivalent antitop mode). When kinematically allowed, $p p \rightarrow t \bar{t} \rightarrow b b H^{-} W^{+}+$c.c. provides the most important source of light charged Higgs bosons, above and beyond the yield of various direct production modes: $g b \rightarrow t H^{+}\left(\right.$or $\left.g g \rightarrow t \bar{b} H^{+}\right)$[4], $g g \rightarrow W^{ \pm} H^{\mp}$, and $b \bar{b} \rightarrow W^{ \pm} H^{\mp}[5], q \bar{q}^{\prime} \rightarrow \phi H^{ \pm}$, where $\phi$ denotes one of the three neutral Higgs bosons [6], $g g \rightarrow H^{+} H^{-}$and $q \bar{q} \rightarrow$ $H^{+} H^{-}$[7], $q b \rightarrow q^{\prime} H^{+} b$ [8], and $c \bar{s}, c \bar{b} \rightarrow H^{+}$[9]. (See also Refs. [10,11] for a review of all available $H^{ \pm}$hadroproduction modes in a $2 \mathrm{HDM}$.)

Assuming a light charged Higgs boson, i.e., such that $M_{H^{ \pm}}<m_{t}-m_{b}$, the ATLAS and CMS experiments have already drawn an exclusion on $\mathrm{BR}\left(t \rightarrow b H^{+}\right) \times$ $\operatorname{BR}\left(H^{ \pm} \rightarrow \tau \nu\right)$ based on the search for the corresponding decay chain $[12,13]$. Other channels, such as $H^{+} \rightarrow c \bar{s}$, have also been searched for by ATLAS and CMS $[14,15]$. Assuming that $\mathrm{BR}\left(H^{+} \rightarrow c \bar{s}\right)=100 \%$, one can set a limit on $\operatorname{BR}\left(t \rightarrow b H^{+}\right)$to be in the range of $5 \%$ to $1 \%$ for a charged Higgs boson mass between 90 and $150 \mathrm{GeV}$. We recall here that charged Higgs bosons have been also searched for at LEP-II using charged Higgs boson pair production followed by either $H^{ \pm} \rightarrow \tau \nu, H^{ \pm} \rightarrow c s$ or $H^{ \pm} \rightarrow W^{ \pm} A$ [15]. If the charged Higgs boson decays dominantly to $\tau \nu$ or $c s$ [16], the LEP-II lower bound on the mass is of the order of $80 \mathrm{GeV}$, while in the case where charged Higgs decay is dominated by $W^{ \pm} A^{0}$, via a light $C P$-odd Higgs state $\left(M_{A^{0}} \sim 12 \mathrm{GeV}\right)$, the lower bound on the charged Higgs mass is about $72 \mathrm{GeV}$ [15].

The aim of this paper is to show that the bosonic decays of a light (i.e., with $72 \mathrm{GeV}<M_{H^{ \pm}}<m_{t}$ ) charged Higgs boson, specifically, $H^{ \pm} \rightarrow W^{ \pm *} h$ and/or $H^{ \pm} \rightarrow W^{ \pm *} A$, in a scenario where $H^{0}$ is the discovered SM-like Higgs state (i.e., $H=H_{\text {obs }}$ ), could be substantial and may compete with the aforementioned fermionic modes over specific regions of the 2HDM Type-I parameter space. Our study builds upon the results of Ref. [17], yet we surpass this paper in several directions. Firstly, we allow for $t \rightarrow b H^{+}$ decays in the presence of both single and double top-quark production (whereas Ref. [17] only considered the latter). Secondly, unlike that reference, which only performed an inclusive analysis, we proceed here to a full MC simulation in the presence of a parton shower, hadronization, heavy flavor decays, and detector effects. Thirdly, we allow here for $A^{0}$ decays into $\tau^{+} \tau^{-}$pairs, wherein the latter can in turn decay fully hadronically, fully leptonically, and semileptonically (or semihadronically), whereas Ref. [17] made no assumption on the $A^{0}$ decay patterns.

The layout of the paper is as follows. In the next section, we describe the 2HDM realisation we are interested in (i.e., a Type-I), whereas, in the following one, we outline the theoretical and experimental constraints acting upon it.
Then, we define the BPs to be used for the MC analysis, which is then described in detail. Following the discussion of the results, we will finally conclude. (We also have two Appendixes, one detailing the performance of a standard cutflow and another illustrating the potential of an improved one, with potentially lower thresholds on some key kinematic variables.)

\section{THE 2HDM TYPE-I}

The 2HDM consists of two complex $\mathrm{SU}(2)_{L}$ (where $L$ indicates the isospin) (pseudo)scalar Higgs doublets $\Phi_{1,2}$ with the same hypercharge $Y_{1,2}=+1 / 2$ that give masses to SM gauge bosons as well as fermions. Explicitly, $\Phi_{1}$ and $\Phi_{2}$ are defined as

$$
\begin{aligned}
& \Phi_{1}=\left(\begin{array}{c}
\phi_{1}^{+} \\
\left(v_{1}+\phi_{1}^{0}+i \chi_{1}^{0}\right) / \sqrt{2}
\end{array}\right) \text { and } \\
& \Phi_{2}=\left(\begin{array}{c}
\phi_{2}^{+} \\
\left(v_{2}+\phi_{2}^{0}+i \chi_{2}^{0}\right) / \sqrt{2}
\end{array}\right) .
\end{aligned}
$$

The 2HDM Lagrangian involving the two Higgs doublets $\Phi_{1}$ and $\Phi_{2}$ can be written as:

$$
\mathcal{L}=\sum_{i}\left|D_{\mu} \Phi_{i}\right|^{2}-V\left(\Phi_{1}, \Phi_{2}\right)+\mathcal{L}_{\text {Yukawa }} .
$$

The first term is the kinetic one for the (pseudo)scalar fields that generate the gauge boson masses as well as the Higgs boson interactions with the gauge bosons themselves. The second term is the scalar potential, and the third one is the Yukawa Lagrangian. The covariant derivative is given by $D_{\mu}=\partial_{\mu}+i g \vec{T}_{a} \vec{W}_{\mu}^{a}+i g^{\prime} \frac{Y_{i}}{2} B_{\mu}$, where $W_{\mu}^{a}$ and $B_{\mu}$ are, respectively, the $\mathrm{SU}(2)_{L}$ and $\mathrm{U}(1)_{Y}$ gauge fields, with $g$ and $g^{\prime}$ the associated gauge coupling constants.

The most general scalar potential, of dimension 4, that is $\mathrm{SU}(2)_{L} \times U(1)_{Y}$ invariant, $C P$ conserving and which possess a $Z_{2}$ symmetry $\left(\Phi_{1} \rightarrow-\Phi_{1}\right.$ or $\left.\Phi_{2} \rightarrow-\Phi_{2}\right)$, which is introduced to avoid flavor changing neutral currents (FCNCs) yet it is softly broken (by a dimension-2 term, the one $\propto m_{12}^{2}$ below) to enable a nontrivial dynamics, is given by

$$
\begin{aligned}
V\left(\Phi_{1}, \Phi_{2}\right)= & m_{11}^{2} \Phi_{1}^{\dagger} \Phi_{1}+m_{22}^{2} \Phi_{2}^{\dagger} \Phi_{2}-m_{12}^{2}\left(\Phi_{1}^{\dagger} \Phi_{2}+\text { H.c. }\right) \\
& +\frac{\lambda_{1}}{2}\left(\Phi_{1}^{\dagger} \Phi_{1}\right)^{2}+\frac{\lambda_{2}}{2}\left(\Phi_{2}^{\dagger} \Phi_{2}\right)^{2} \\
& +\lambda_{3}\left(\Phi_{1}^{\dagger} \Phi_{1}\right)\left(\Phi_{2}^{\dagger} \Phi_{2}\right)+\lambda_{4}\left(\Phi_{1}^{\dagger} \Phi_{2}\right)\left(\Phi_{2}^{\dagger} \Phi_{1}\right) \\
& +\frac{\lambda_{5}}{2}\left[\left(\Phi_{1}^{\dagger} \Phi_{2}\right)^{2}+\text { H.c. }\right],
\end{aligned}
$$

where all parameters are real valued. After spontaneous electroweak symmetry breaking (EWSB) of $\mathrm{SU}(2)_{L} \times$ $U(1)_{Y}$ down to $\mathrm{U}(1)_{\mathrm{EM}}$ [where $L$ indicates the electromagnetic (EM) group], each doublet obtains a vacuum expectation value $(\mathrm{VEV}) v_{i}(i=1,2)$, such that they can 
be fixed from the EW scale through the relation $v=\sqrt{v_{1}^{2}+v_{2}^{2}}=246 \mathrm{GeV}$. Furthermore, upon EWSB, three Goldstone bosons $\left(G^{ \pm}\right.$and $\left.G\right)$ are absorbed as the longitudinal component of the gauge bosons $\left(W^{ \pm}\right.$and $Z$, respectively) so that the latter gain their mass. The remaining five degrees of freedom (out of the initial eight pertaining to the two complex doublets) become the physical Higgs bosons, namely, the aforementioned $h, H, A$, and $H^{ \pm}$.

From the two minimization conditions of the 2HDM, one can eliminate $m_{11}^{2}$ and $m_{22}^{2}$ in favor of other (pseudo) scalar inputs so that we are then left with the following seven free independent real parameters:

$\tan \beta\left(\equiv \frac{v_{2}}{v_{1}}\right), \quad m_{12}^{2}, \quad \lambda_{1}, \quad \lambda_{2}, \quad \lambda_{3}, \quad \lambda_{4}, \quad \lambda_{5}$.

Instead of the eight parameters of Eq. (4), a more convenient choice would be:

$$
M_{h^{0}}, \quad M_{H^{0}}, \quad M_{A^{0}}, \quad M_{H^{ \pm}}, \quad \alpha, \quad \tan \beta, \quad m_{12}^{2},
$$

where $\alpha$ is the mixing angle that rotates the nonphysical states $\phi_{1}^{0}$ and $\phi_{2}^{0}$ to the physical ones $h^{0}$ and $H^{0}$.

For the Yukawa Lagrangian, if we proceed like in the SM and ask that both Higgs doublets couple to all SM fermions, we end up with neutral Higgs couplings to fermions that are flavor violating, i.e., the aforementioned FCNCs. The existence of such interactions would induce large contributions to low energy observables such as $B, D$, and $K$ meson oscillations and others, which could contradict precise experimental measurements. To evade this problem, one can advocate so-called natural flavor conservation (NFC) by imposing the aforementioned discrete symmetry, $Z_{2}$ [18], which forbids them. As intimated, to achieve a 2HDM dynamics at once compliant with EWSB and FCNC limits yet offering a (pseudo)scalar mass spectrum that is experimentally interesting, we allow for a soft $Z_{2}$ breaking (i.e., a small $m_{12}^{2}$ term).

There exist several ways of coupling the two Higgs doublets to the SM fermions. In the 2HDM Type-I, $\Phi_{2}$ couple to all fermions, just like in the SM, while the other $\Phi_{1}$ does not. ${ }^{1}$ The discrete symmetry behind this assignment is $\Phi_{1} \rightarrow-\Phi_{1}$. The Yukawa Lagrangian of the Type-I model is

$$
\mathcal{L}_{\text {Yukawa }}=Y_{d} \bar{Q}_{L} \Phi_{2} d_{R}+Y_{u} \bar{Q}_{L} \tilde{\Phi}_{2} u_{R}+Y_{e} \bar{L}_{L} \Phi_{2} e_{R}+\text { H.c. },
$$

where $\bar{Q}_{L}$ and $\bar{L}_{L}$ are the left-handed quark and lepton doublets, $d_{R}, u_{R}$, and $e_{R}$ are the right-handed up-type quark, down-type quark, and lepton singlets, respectively, $Y_{u}, Y_{d}$, and $Y_{e}$ are the corresponding Yukawa coupling

\footnotetext{
${ }^{1}$ See [19] for a review of the 2HDM covering other Yukawa types.
}

TABLE I. Yukawa couplings in the 2HDM Type-I, wherein $c$ and $s$ stand for cos and sin, respectively.

\begin{tabular}{lcccccccc}
\hline$\kappa_{u}^{h}$ & $\kappa_{d}^{h}$ & $\kappa_{l}^{h}$ & $\kappa_{u}^{H}$ & $\kappa_{d}^{H}$ & $\kappa_{l}^{H}$ & $\kappa_{u}^{A}$ & $\kappa_{d}^{A}$ & $\kappa_{l}^{A}$ \\
\hline$c_{\alpha} / s_{\beta}$ & $c_{\alpha} / s_{\beta}$ & $c_{\alpha} / s_{\beta}$ & $s_{\alpha} / s_{\beta}$ & $s_{\alpha} / s_{\beta}$ & $s_{\alpha} / s_{\beta}$ & $c_{\beta} / s_{\beta}$ & $-c_{\beta} / s_{\beta}$ & $-c_{\beta} / s_{\beta}$ \\
\hline \hline
\end{tabular}

matrices, and $\tilde{\Phi}_{2}=i \sigma_{2} \Phi_{2}^{*}$ (where $\sigma_{2}$ is the second Pauli matrix). After expressing the weak eigenstates of $\Phi_{2}$ in terms of the physical ones, the Yukawa Lagrangian in Eq. (6) becomes:

$$
\begin{aligned}
- & \mathcal{L}_{\text {Yukawa }} \\
= & \sum_{\psi=u, d, l}\left(\frac{m_{\psi}}{v} \kappa_{\psi}^{h} \bar{\psi} \psi h^{0}+\frac{m_{\psi}}{v} \kappa_{\psi}^{H} \bar{\psi} \psi H^{0}-i \frac{m_{\psi}}{v} \kappa_{\psi}^{A} \bar{\psi} \gamma_{5} \psi A^{0}\right) \\
& +\left(\frac{V_{u d}}{\sqrt{2} v} \bar{u}\left(m_{u} \kappa_{u}^{A} P_{L}+m_{d} \kappa_{d}^{A} P_{R}\right) d H^{+}\right. \\
& \left.+\frac{m_{l} \kappa_{l}^{A}}{\sqrt{2} v} \bar{\nu}_{L} l_{R} H^{+}+\text {H.c. }\right)
\end{aligned}
$$

where $\kappa_{i}^{S}$ are the Yukawa couplings in the 2HDM. The values of those couplings in the 2HDM Type-I are given in Table I.

From the kinetic terms of the Higgs doublets, one can derive the interactions between the gauge bosons and a pair of Higgs (pseudo)scalars, such as $A^{0} h^{0} Z^{0}, H^{ \pm} h^{0} W^{ \pm}$, $H^{ \pm} A^{0} W^{ \pm}$, and $H^{ \pm} H^{0} W^{ \pm}$. The corresponding couplings are determined by the gauge group structure as well as the angles $\alpha$ and $\beta$ [20]. They are given by the following relations:

$$
\begin{gathered}
g_{H^{ \pm} h^{0} W^{ \pm}}=\frac{g \cos (\beta-\alpha)}{2}\left(p_{h^{0}}-p_{H^{ \pm}}\right)^{\mu}, \\
g_{H^{ \pm} H^{0} W^{ \pm}}=\frac{g \sin (\beta-\alpha)}{2}\left(p_{h^{0}}-p_{H^{ \pm}}\right)^{\mu}, \\
g_{H^{ \pm} A^{0} W^{ \pm}}=\frac{g}{2}\left(p_{A^{0}}-p_{H^{ \pm}}\right)^{\mu}, \\
g_{A^{0} h^{0} Z^{0}}=\frac{g \sin (\beta-\alpha)}{2}\left(p_{h^{0}}-p_{A^{0}}\right)^{\mu},
\end{gathered}
$$

with $p_{\mu}$ being the incoming momentum for the corresponding particle.

If the charged Higgs boson is light, the top (anti)quark can decay into either $b W^{ \pm}$or $b H^{ \pm}$. The first decay is controlled by a SM gauge coupling, while the second decay depends upon $\beta$, and it can be enhanced for small $\tan \beta$. Conventionally, a light charged Higgs boson is assumed to decay into either $\tau \nu$ or $c s$, with the corresponding couplings given in Table I. However, if there is an additional light neutral Higgs boson $h^{0}$ or $A^{0}$, the additional decay channels into $A^{0} W^{ \pm(*)}$ and $h^{0} W^{ \pm(*)}$ would open up, wherein the $W^{ \pm}$ boson can be on or off shell, depending on the mass differences $M_{H^{ \pm}}-M_{A^{0}}$ and $M_{H^{ \pm}}-M_{h^{0}}$, respectively. The $H^{ \pm} \rightarrow h^{0} W^{ \pm(*)}$ channel for a light charged Higgs boson is 
TABLE II. 2HDM parameter ranges adopted for our scan. (Note that we have taken $M_{H^{0}}=125 \mathrm{GeV}$.)

\begin{tabular}{lc}
\hline \hline Parameters & Ranges \\
\hline$M_{h^{0}}$ & {$[10,120] \mathrm{GeV}$} \\
$M_{A^{0}}$ & {$[10,120] \mathrm{GeV}$} \\
$M_{H^{ \pm}}$ & {$[80,170] \mathrm{GeV}$} \\
$\sin (\beta-\alpha)$ & {$[-0.3,-0.05]$} \\
$m_{12}^{2}$ & {$\left[0, M_{H^{0}}^{2} \sin \beta \cos \beta\right] \mathrm{GeV}^{2}$} \\
$\tan \beta$ & {$[1,60]$} \\
\hline \hline
\end{tabular}

open only if we demand that $H^{0}$ is the observed $125 \mathrm{GeV}$ SM-like Higgs state (which we do here). In this case, $|\cos (\beta-\alpha)| \sim 1$ is preferred by experiments, and thus, the $H^{ \pm} h^{0} W^{ \pm}$coupling is large. The $H^{ \pm} A^{0} W^{ \pm}$coupling is independent of $\sin (\beta-\alpha)$ and thus, always large. Finally, the $H^{ \pm} \rightarrow H^{0} W^{ \pm(*)}$ decay channel is greatly suppressed since it is proportional to $|\sin (\beta-\alpha)|^{2}$, and the mass difference involved $\left(M_{H^{ \pm}}-M_{H^{0}}\right)$ could be very small since $M_{H^{ \pm}}<m_{t}-m_{b}$ and $M_{H^{0}}=125 \mathrm{GeV}$.

To study our 2HDM Type-I, we perform a systematic numerical scan over its parameter space as illustrated in Table II. During the scan, each sampled point is subject to a set of theoretical and experimental constraints, which are described in the following section.

\section{THEORETICAL AND EXPERIMENTAL CONSTRAINTS}

The 2HDM parameters in Eq. (5) are constrained by a number of theoretical considerations, such as vacuum stability [21], perturbativity, perturbative unitarity [22], and experimental limits from LEP, Tevatron, LHC, as well as $B$ physics experiments. We list here the constraints that we have used.

From the theoretical side, we have the following:

(i) A necessary condition for the stability of the vacuum comes from requiring that the scalar potential remains bounded from below when the (pseudo) scalar fields become large. This should be fulfilled in any arbitrary direction in the field space. In the limit of large field values, the scalar potential is dominated by quartic couplings, and one can show that the tree-level vacuum stability constraints are [21]

$$
\begin{gathered}
\lambda_{1}>0, \quad \lambda_{2}>0, \quad \lambda_{3}>-\sqrt{\lambda_{1} \lambda_{2}}, \\
\lambda_{3}+\lambda_{4}-\left|\lambda_{5}\right|>-\sqrt{\lambda_{1} \lambda_{2}} .
\end{gathered}
$$

(ii) We require that all $\lambda_{i}$ 's remain perturbative and satisfy $\left|\lambda_{i}\right| \leq 8 \pi$.

(iii) Perturbative unitarity constraints [22] are those obtained by requesting that the $S$-wave component of the various (pseudo)scalar-(pseudo)scalar, (pseudo)scalar-gauge boson, and gauge-gauge bosons scatterings remain unitary at high energy. Such a condition implies a set of constraints that have to be fulfilled and are given in [22].

(iv) We also check the consistency at $95 \%$ confidence level (CL) with the experimental measurements of the oblique parameters $S, T$, and $U$. We compare those to the fit values of [23], i.e., $S=0.05 \pm 0.11$, $T=0.09 \pm 0.13$, and $U=0.01 \pm 0.11$.

Note that unitarity, perturbativity, vacuum stability, as well as $S, T$, and $U$ constraints, are enforced through the 2HDMC public code [24].

From the experimental side, we considered the following.

(i) $B$-physics observables are implemented with the code SuperIso v4.0 [25]. Specifically, we have used the following measurements:
(1) $\left.\mathrm{BR}\left(B \rightarrow X_{s} \gamma\right)\right|_{E_{\gamma}>1.6 \mathrm{GeV}}=(3.32 \pm 0.3) \times 10^{-4}$ $[26,27]$,
(2) $\operatorname{BR}\left(B_{s} \rightarrow \mu \mu\right)=(3.1 \pm 1.4) \times 10^{-9}[26,28]$,
(3) $\operatorname{BR}\left(B^{+} \rightarrow \tau^{+} \nu_{\tau}\right)=\left(1.06_{-0.28}^{+0.38}\right) \times 10^{-4}[26,29]$.

For all such observables, we allow a $2 \sigma$ tolerance from the above measurements.

(ii) Consistency with the $Z$ width measurement from LEP, $\Gamma_{Z}=2.4952 \pm 0.0023 \mathrm{GeV}$ [30]. Specifically, the partial width $\Gamma\left(Z \rightarrow h^{0} A^{0}\right)$ was required to fall within the $2 \sigma$ experimental uncertainty of the measurement $(\leq 4.6 \mathrm{MeV})$.

(iii) The indirect constraints from Higgs data measurements are taken into account by using HiggsSignal (version 2.2.0) public code [31,32], which includes both Run-1 data [3] from the combined ATLAS and CMS analysis at $\sqrt{s}=7$ and $8 \mathrm{TeV}$, and results from Run 2 at $\sqrt{s}=13 \mathrm{TeV}$ up to $36 \mathrm{fb}^{-1}$ [33-39] from ATLAS, as well as from CMS [40-49]. Assuming no deviations from SM predictions. We require that the relevant quantities, calculated with HiggsSignals, satisfy $\Delta \chi^{2}=\chi^{2}-\chi_{\min }^{2}$ for these measurements at $95 \% \mathrm{CL}\left(\Delta \chi^{2}<5.99\right)$.

(iv) Consistency of all Higgs states with the direct search constraints from LEP, Tevatron, and LHC at the 95\% CL, which are tested using the updated version of HiggsBounds-5.3.2beta [50] in default configuration.

\section{NUMERICAL RESULTS OF THE SCAN AND BENCHMARK POINTS}

In this section, we present the results of the aforementioned scans and propose six BPs ameanable to $\mathrm{MC}$ analysis. In the scan, we restrict ourselves to negative $\sin (\beta-\alpha) .^{2}$ This parameter space has been studied before in Ref. [51], where neutral Higgs bosons $A^{0}$ and $h^{0}$ could

\footnotetext{
${ }^{2} \mathrm{We}$ remind the reader here that the fermionic couplings of the $h^{0}$ boson are proportional to $\cos \alpha / \sin \beta=\sin (\beta-\alpha)+$ $\cot \beta \cos (\beta-\alpha)$; hence, they will be reduced for negative $\sin (\beta-\alpha)$, and therefore LEP limits are easily satisfied even for the light $h^{0}$ state considered in this study.
} 
be lighter than $M_{H^{0}} / 2$. In the current work, we extend the work [51] by examining the detailed properties in this specific parameter space. We will mainly focus on the search potential of the LHC to this parameter space.

BPs are given in Table (III), which can serve as a new physics search not only for the LHC but also for the future $e^{+} e^{-}$colliders. At the future $e^{+} e^{-}$colliders (say Higgs factories with collision energy $\sqrt{s}=250 \mathrm{GeV}$ ), these BPs can be discovered either via $e^{+} e^{-} \rightarrow h^{0} A^{0}$ or $e^{+} e^{-} \rightarrow$ $H^{+} H^{-}$due to their light masses. Production rates and the search modes for each of these six BPs will be different due to different mass spectra and different decay branching fractions of $h^{0}$ and $A^{0}$ and $H^{ \pm}$as well. To discover these processes at the future $e^{+} e^{-}$colliders, the dominant background will be $e^{+} e^{-} \rightarrow W^{+} W^{-}$and $e^{+} e^{-} \rightarrow t \bar{t}$ (when the collision energy is larger than $350 \mathrm{GeV}$ ). The detailed analysis can be done in the future.

\section{A. Some essential features of light charged Higgs bosons in the 2HDM Type-I}

We first illustrate the BRs of $h^{0}, A^{0}, H^{ \pm}$, and $t$ into different final states. We start with Fig. 1. In the top two frames, we present $\operatorname{BR}\left(h^{0} / A^{0} \rightarrow \tau^{+} \tau^{-}\right)$(left) and
$\mathrm{BR}\left(h^{0} / A^{0} \rightarrow b \bar{b}\right)$ (right). Before discussing these, though, we remind the reader that in the 2HDM Type-I, the $A^{0}$ couplings to fermions have all the same $\tan \beta$ dependence. Therefore, when all bosonic decays are not open, the BRs of the $A^{0}$ state into fermions will not depend on $\tan \beta$. In the case of the $h^{0}$ state, however, things are different because of the presence of the $h^{0} \rightarrow V V^{*}\left(V=W^{ \pm}, Z\right)$ decay channels. Nonetheless, in the exact alignment limit (from which we do not depart significantly), where the coupling $h^{0} V V$ vanishes, the decay phenomenology of the $h^{0}$ state follows closely that of the $A^{0}$ one.

From the aforementioned figure, it is clear that the $\operatorname{BR}\left(h^{0} / A^{0} \rightarrow \tau^{+} \tau^{-}\right)$could reach at best $8 \%$, while $\operatorname{BR}\left(h^{0} / A^{0} \rightarrow b \bar{b}\right)$ can reach up to $80 \%$. However, the former final state is much cleaner than the latter one in the LHC environment. In this connection, upon recalling that the full decay chain $H^{ \pm} \rightarrow h^{0} / A^{0} W^{ \pm(*)} \rightarrow b \bar{b} W^{ \pm(*)}$ is subject to interference effects with $H^{ \pm} \rightarrow b t^{*} \rightarrow b \bar{b} W^{ \pm(*)}$ and significant background from $t \bar{t}$ production and decay [52-54], we are induced to elect as a tentative signal in our analysis the case of $\tau$ final states. Furthermore, in the bottom-left frame of Fig. 1, we demonstrate that the bosonic decays of the charged Higgs boson,
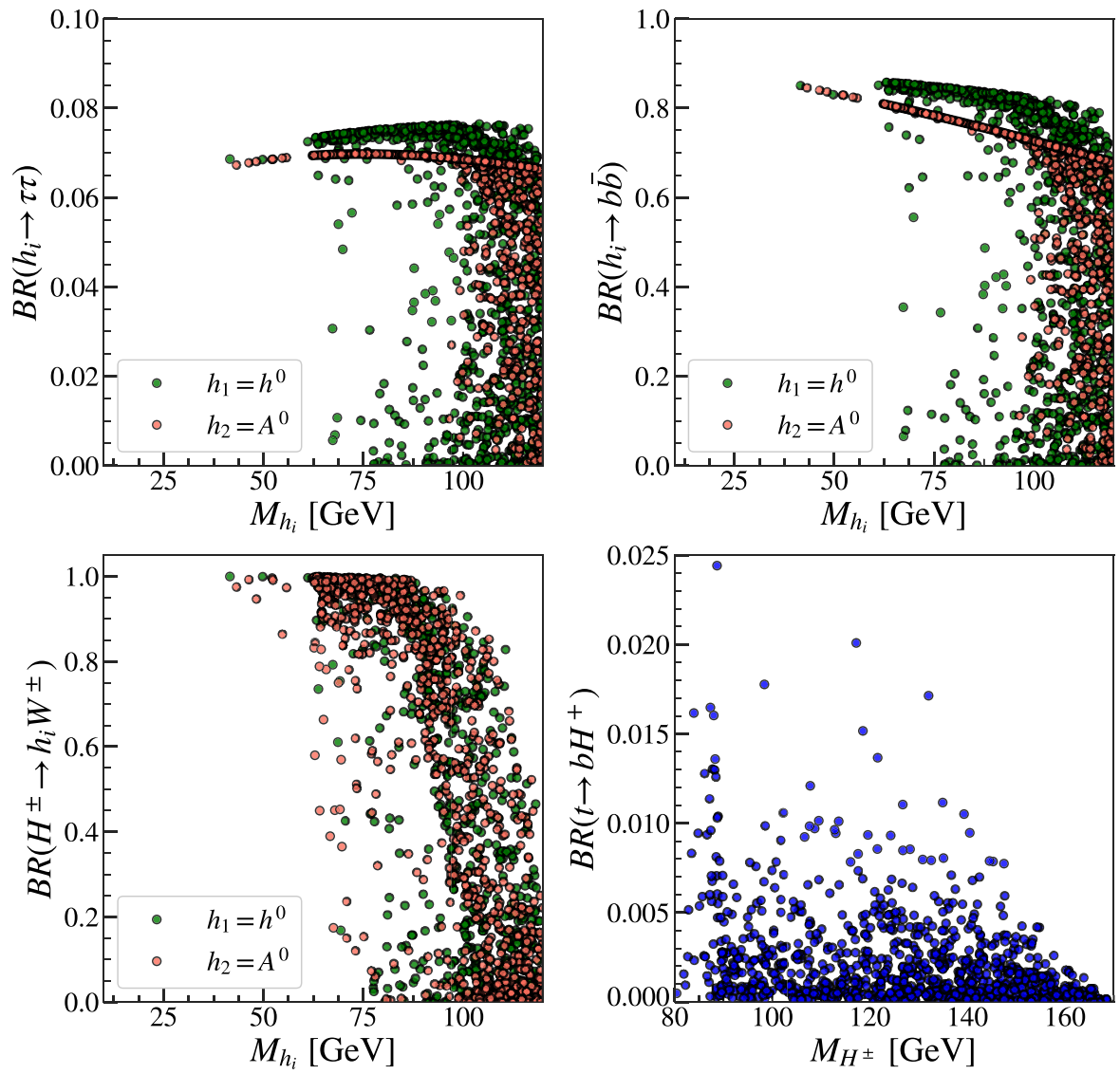

FIG. 1. (Top left) $\mathrm{BR}\left(h^{0} / A^{0} \rightarrow \tau^{+} \tau^{-}\right)$. (Top right) $\mathrm{BR}\left(h^{0} / A^{0} \rightarrow b \bar{b}\right)$. (Bottom left) $\mathrm{BR}\left(H^{ \pm} \rightarrow h^{0} / A^{0} W^{ \pm(*)}\right)$. (Bottom right) $\operatorname{BR}\left(t \rightarrow b H^{+}\right)$. BRs are given as a function of the relevant Higgs masses. 

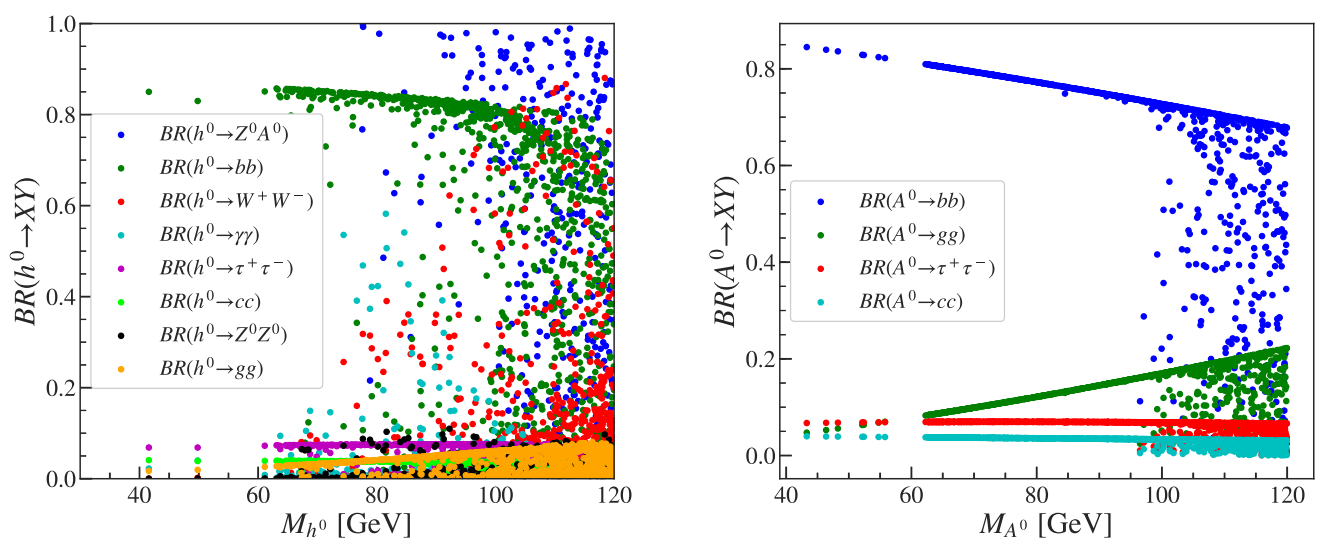

FIG. 2. BRs of $h^{0}$ (left) and $A^{0}$ (right) as a function of its mass.

$\operatorname{BR}\left(H^{ \pm} \rightarrow h^{0} / A^{0} W^{ \pm(*)}\right)$, could become the dominant ones, even reaching $100 \%$ in some cases (see also [17]). Finally, in the bottom-right frame of Fig. 1, we present $\mathrm{BR}\left(t \rightarrow b H^{+}\right)$, illustrating the fact that this decay channel of the top (anti)quark is not excessively suppressed with respect to the SM one $t \rightarrow b W^{+}$. We therefore recommend the $t \rightarrow b H^{ \pm} \rightarrow b h^{0} / A^{0} W^{ \pm(*)} \rightarrow b \tau^{+} \tau^{-} W^{ \pm(*)}$ decay chain as the one to be pursued experimentally, assuming either single or double top (anti)top hadro-production.

A further advantage of the $\tau^{+} \tau^{-}$decay is that its rate remains substantial as a function of the $h^{0}$ and $A^{0}$ masses when the latter are below $100 \mathrm{GeV}$ or so, as seen in Fig. 2. This implies that there is little loss of sensitivity through the $\tau^{+} \tau^{-}$mode in an experimental search, no matter the actual value of $M_{h^{0}}$ or $M_{A^{0}}$ in our mass ranges of interest. However, other $h^{0}$ and/or $A^{0}$ decay modes might be useful. In fact, one can see from Fig. 2 (left) that $h^{0}$ could become fermiophobic in some cases, making $\mathrm{BR}\left(h^{0} \rightarrow \gamma \gamma\right)$ [51,55] or $\mathrm{BR}\left(h^{0} \rightarrow Z A^{0}\right)$ the dominant decay mode. Further, from
Fig. 2 (right), it is noteworthy that there are points where the suppression of $A^{0} \rightarrow b \bar{b}$ for large $\tan \beta$ value allows for a substantial enhancement of $A^{0} \rightarrow Z h^{0}$ and/or $A^{0} \rightarrow W^{ \pm(*)} H^{\mp}$. It might also be interesting to study these alternative decay modes and their sensitivity at the LHC.

To quantify the size of the light charged Higgs cross section from the single and double top (anti)quark production followed by the discussed decay chains, we evaluate the quantities $X^{t j}\left(h_{i}, f \bar{f}\right)$ and $X^{t t}\left(h_{i}, f \bar{f}\right)$, respectively, which are defined as follows:

$$
\begin{aligned}
X^{t j}\left(h_{i}, f \bar{f}\right)= & \sigma(p p \rightarrow t j) \times \mathrm{BR}\left(t \rightarrow b H^{+}\right) \\
& \times \mathrm{BR}\left(H^{+} \rightarrow h_{i} W^{+}\right) \times \mathrm{BR}\left(h_{i} \rightarrow f \bar{f}\right), \\
X^{t t}\left(h_{i}, f \bar{f}\right)= & \sigma(p p \rightarrow t t) \times \mathrm{BR}\left(t \rightarrow b H^{+}\right) \\
& \times \mathrm{BR}\left(t \rightarrow b W^{+}\right) \times \mathrm{BR}\left(H^{+} \rightarrow h_{i} W^{+}\right) \\
& \times \mathrm{BR}\left(h_{i} \rightarrow f \bar{f}\right) .
\end{aligned}
$$

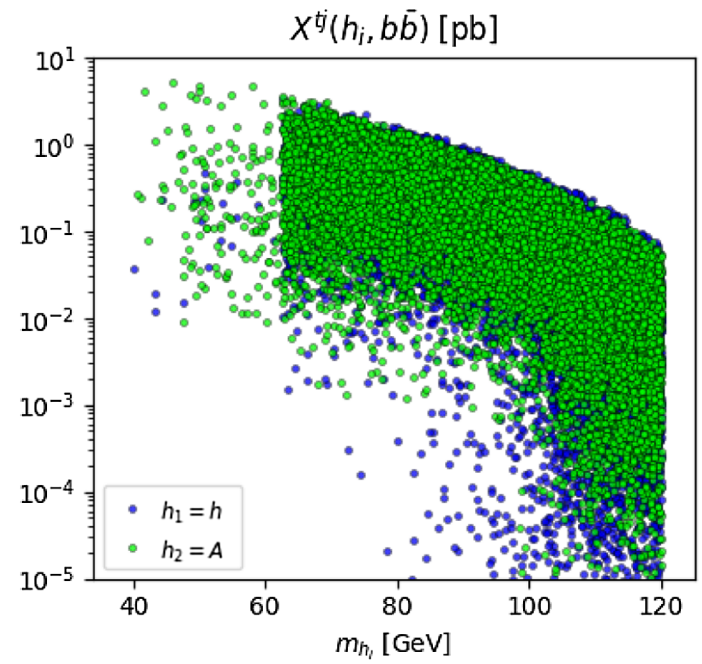

FIG. 3. Cross section for $\sigma(p p \rightarrow t j) \times \mathrm{BR}\left(t \rightarrow b H^{ \pm}\right) \times \mathrm{BR}\left(H^{ \pm} \rightarrow h_{i} W^{ \pm}\right) \times \mathrm{BR}\left(h_{i} \rightarrow f f\right)$ as a function of the $h^{0}$ and $A^{0}$ mass with $f f=\tau^{+} \tau^{-}$(left) and $f f=b \bar{b}$ (right). 

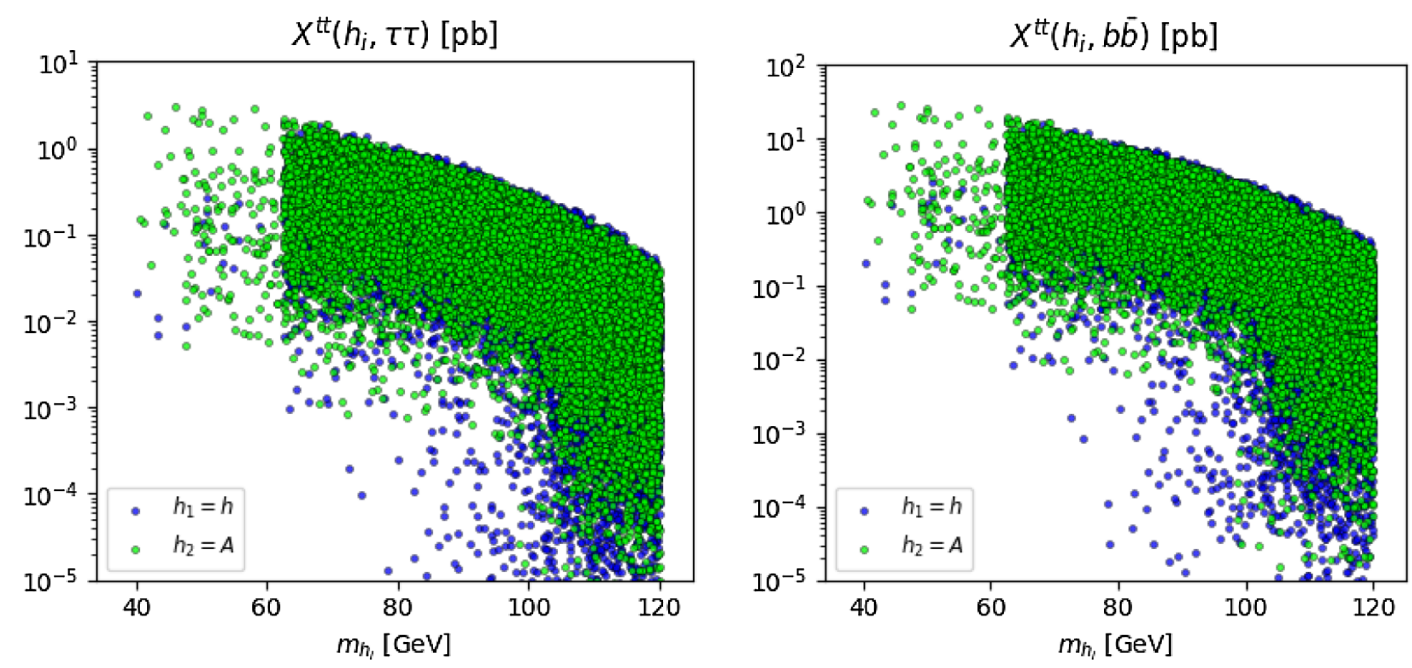

FIG. 4. Cross section $\sigma(p p \rightarrow t \bar{t}) \times \mathrm{BR}\left(t \rightarrow b H^{ \pm}\right) \times \mathrm{BR}\left(t \rightarrow b W^{ \pm}\right) \times \mathrm{BR}\left(H^{ \pm} \rightarrow h_{i} W^{ \pm}\right) \times \mathrm{BR}\left(h_{i} \rightarrow f f\right)$ as a function of the $h^{0}$ and $A^{0}$ mass with $f \bar{f}=\tau^{+} \tau^{-}$(left) and $f \bar{f}=b \bar{b}$ (right).

(Notice that the subprocesses contributing to $t j$ production are $b q \rightarrow t q^{\prime}$ and $q \bar{q}^{\prime} \rightarrow t b$, while those entering $t \bar{t}$ production are $q \bar{q}, g g \rightarrow t \bar{t}$.)

In Figs. 3 and 4, we give the numerical results from our scan for both $X^{t j}\left(h_{i}, f \bar{f}\right)$ and $X^{t t}\left(h_{i}, f \bar{f}\right)$, respectively, for $h^{0}$ and $A^{0}$ decaying into $\tau^{+} \tau^{-}$(left frames) or $b \bar{b}$ (right frames), i.e., $f f=\tau^{+} \tau^{-}$and $f f=b \bar{b}$ in turn. There is an obvious feature in Figs. 3 and 4, i.e., that when the mass of $M_{h^{0}}$ or $M_{A^{0}}$ is smaller than half the SM-like Higgs boson $M_{H^{0}}=125 \mathrm{GeV}$, significantly fewer points are allowed, which can be attributed to the constraints on the decay $H^{0} \rightarrow h^{0} h^{0}$ affecting the SM-like Higgs boson width. While the bottom decay rates are dominant over the tau ones, it is clear that the production of charged Higgs bosons from the sum of single and double (anti)top quark decays followed by either of our bosonic modes could reach more than a few $\mathrm{pb}$ in the $\tau^{+} \tau^{-}$channel. While this value is handsomely large at the inclusive level, it should be recalled that the $W^{ \pm(*)}$ boson emerging from the $H^{ \pm} \rightarrow$ $h^{0} / A^{0} W^{ \pm(*)}$ decay would be searched for in its leptonic transitions, i.e., $W^{ \pm(*)} \rightarrow l^{ \pm} \nu$, which are of order $20 \%$ only, so this calls for using all possible decay patterns of the $\tau^{+} \tau^{-}$ pair, i.e., fully hadronic, fully leptonic, and semileptonic (or semihadronic), in order to maximize experimental sensitivity. These three signatures are therefore what we will pursue in our MC study.

\section{B. Benchmark points}

In order to perform our MC simulation, out of our scan, we select six BPs. These six BPs can be categorized into two groups: the first group with $M_{h^{0}}<M_{A^{0}}$ and the second group with $M_{A^{0}}<M_{h^{0}}$. In each group, there are three BPs. For the MC study for the LHC, three BPs for each group are supposed to reveal which factors (masses and decay modes) are more important and crucial to determine the sensitivity.
Detailed information of these six BPs, including mass spectra and decay BRs, is presented in Table III. There are several salient features of these BPs that is worth dwelling upon.

(i) Both the extra neutral Higgs bosons, $A^{0}$ and $h^{0}$, are lighter than the discovered Higgs boson, $H^{0}$. (Notice that some BPs have the $h^{0} \rightarrow Z A^{0}$ and/or $A^{0} \rightarrow Z h^{0}$ decay channels open, so they will be competing with $h^{0}, A^{0} \rightarrow \tau^{+} \tau^{-}$decays.)

(ii) The charged Higgs boson has a mass smaller than the $t$ mass, but the sum of its mass with the mass of an extra neutral Higgs boson (either $A^{0}$ or $h^{0}$ ) is larger than the top quark mass, except for BP6.

(iii) The rates for $\operatorname{BR}\left(t \rightarrow b H^{ \pm}\right) \times \operatorname{BR}\left(H^{ \pm} \rightarrow \tau^{ \pm} \nu_{\tau}\right)$ are less than $12 \times 10^{-4}$, i.e., below the current LHC bound [56].

(iv) The $\operatorname{BR}\left(H^{ \pm} \rightarrow h^{0} W^{ \pm(*)}\right)$ for the first three BPs is equal to $89.9 \%, 83.5 \%$, and $87.7 \%$, respectively, while the $\operatorname{BR}\left(H^{ \pm} \rightarrow A^{0} W^{ \pm(*)}\right)$ for the second three BPs is equal to $93.6 \%, 92.8 \%$, and $83.2 \%$, respectively. Thus, they are very close to their best possible values (recall Fig. 1), in turn, implying that these are amongst the 2HDM Type-I parameter points most accessible to a future LHC analysis. Meanwhile, the $h^{0}\left(A^{0}\right)$ mass and the mass differences between the $H^{ \pm}$and the $h^{0}\left(A^{0}\right)$ are specially chosen to produce different $\tau$ and off-shell $W$ bosons energy in six BPs, which will lead to the different detector responses as shown in the following collider analysis.

We will now proceed to a MC version of the latter.

\section{COLLIDER ANALYSIS}

In this part, we perform a detector level MC simulation to establish the LHC sensitivity to the BPs given in Table III. Due to the kinematics of these BPs, we will focus on the 
TABLE III. The full description of our BPs we take $m_{t}=173.5 \mathrm{GeV}$.

\begin{tabular}{|c|c|c|c|c|c|c|}
\hline Parameters & BP1 & BP2 & BP3 & BP4 & BP5 & BP6 \\
\hline \multicolumn{7}{|c|}{ 2HDM inputs. The Higgs masses are in $\mathrm{GeV}$. } \\
\hline$M_{h^{0}}$ & 80.772 & 78.284 & 85.003 & 115.16 & 119.21 & 111.13 \\
\hline$M_{H^{0}}$ & 125 & 125 & 125 & 125 & 125 & 125 \\
\hline$M_{A^{0}}$ & 116.34 & 116.14 & 109.45 & 64.547 & 72.896 & 62.679 \\
\hline$M_{H^{ \pm}}$ & 124.29 & 112.8 & 132.6 & 117.23 & 132.05 & 98.4 \\
\hline $\sin (\beta-\alpha)$ & -0.073282 & -0.065491 & -0.077173 & -0.13305 & -0.086473 & -0.057954 \\
\hline$\lambda_{6}$ & 0 & 0 & 0 & 0 & 0 & 0 \\
\hline$\lambda_{7}$ & 0 & 0 & 0 & 0 & 0 & 0 \\
\hline$m_{12}^{2}$ & 1045.1 & 676.32 & 1244.7 & 2743.6 & 1979.6 & 2120.2 \\
\hline $\tan \beta$ & 5.5795 & 6.543 & 5.1575 & 4.233 & 3.5324 & 5.6988 \\
\hline \multicolumn{7}{|c|}{ Total decay width in $\mathrm{GeV}$} \\
\hline$\Gamma\left(h^{0}\right)$ & $2.465 \times 10^{-5}$ & $1.6301 \times 10^{-5}$ & $3.1343 \times 10^{-5}$ & 0.0001354 & 0.00019498 & 0.0001218 \\
\hline$\Gamma\left(H^{0}\right)$ & 0.0044033 & 0.0043866 & 0.0044133 & 0.0044791 & 0.0044727 & 0.0043936 \\
\hline$\Gamma\left(A^{0}\right)$ & 0.00013585 & 0.00011075 & 0.00012937 & 0.00010722 & 0.0001734 & $5.752 \times 10^{-5}$ \\
\hline$\Gamma\left(H^{+}\right)$ & 0.00015084 & $5.1564 \times 10^{-5}$ & 0.0002483 & 0.00034353 & 0.00068742 & $5.5684 \times 10^{-5}$ \\
\hline$\Gamma(t)$ & 1.3784 & 1.3789 & 1.3765 & 1.3936 & 1.3894 & 1.3903 \\
\hline \multicolumn{7}{|c|}{$\mathrm{BR}\left(h^{0} \rightarrow X Y\right)$ in $\%$} \\
\hline $\mathrm{BR}\left(h^{0} \rightarrow \tau^{+} \tau^{-}\right)$ & 7.5297 & 7.5114 & 7.5603 & 1.7983 & 4.8386 & 2.5942 \\
\hline $\mathrm{BR}\left(h^{0} \rightarrow g g\right)$ & 4.1758 & 3.9511 & 4.5778 & 1.8932 & 5.4377 & 2.5543 \\
\hline $\mathrm{BR}\left(h^{0} \rightarrow Z^{0} A^{0}\right)$ & $\ldots$ & $\ldots$ & $\ldots$ & 72.548 & 35.026 & 65.059 \\
\hline $\mathrm{BR}\left(h^{0} \rightarrow W^{+} W^{-}\right)$ & 0.029144 & 0.026408 & 0.042102 & 3.4713 & 1.6768 & 0.42731 \\
\hline $\mathrm{BR}\left(h^{0} \rightarrow c c\right)$ & 3.8246 & 3.8413 & 3.7981 & 0.84822 & 2.2665 & 1.2324 \\
\hline $\mathrm{BR}\left(h^{0} \rightarrow b b\right)$ & 84.271 & 84.535 & 83.842 & 18.872 & 50.46 & 27.402 \\
\hline \multicolumn{7}{|c|}{$\operatorname{BR}\left(H^{0} \rightarrow X Y\right)$ in $\%$} \\
\hline $\mathrm{BR}\left(H^{0} \rightarrow \tau^{+} \tau^{-}\right)$ & 5.9984 & 5.9905 & 6.0031 & 6.0389 & 6.0262 & 5.9884 \\
\hline $\mathrm{BR}\left(H^{0} \rightarrow g g\right)$ & 7.3793 & 7.3696 & 7.3851 & 7.4292 & 7.4135 & 7.367 \\
\hline $\mathrm{BR}\left(H^{0} \rightarrow Z^{0} Z^{0}\right)$ & 2.3825 & 2.3942 & 2.3757 & 2.3132 & 2.3406 & 2.3926 \\
\hline $\mathrm{BR}\left(H^{0} \rightarrow W^{+} W^{-}\right)$ & 19.073 & 19.166 & 19.019 & 18.518 & 18.737 & 19.154 \\
\hline $\mathrm{BR}\left(H^{0} \rightarrow c c\right)$ & 2.7834 & 2.7797 & 2.7855 & 2.8022 & 2.7963 & 2.7787 \\
\hline $\mathrm{BR}\left(H^{0} \rightarrow b b\right)$ & 62.016 & 61.934 & 62.065 & 62.435 & 62.304 & 61.913 \\
\hline \multicolumn{7}{|c|}{$\operatorname{BR}\left(A^{0} \rightarrow X Y\right)$ in $\%$} \\
\hline $\mathrm{BR}\left(A^{0} \rightarrow \tau^{+} \tau^{-}\right)$ & 5.6976 & 5.0731 & 6.5871 & 6.9509 & 6.973 & 6.9414 \\
\hline $\mathrm{BR}\left(A^{0} \rightarrow g g\right)$ & 18.133 & 16.1 & 18.93 & 8.7517 & 10.538 & 8.3672 \\
\hline $\operatorname{BR}\left(A^{0} \rightarrow Z^{0} h^{0}\right)$ & 14.745 & 24.117 & 2.6261 & $\ldots$ & $\ldots$ & $\ldots$ \\
\hline $\mathrm{BR}\left(A^{0} \rightarrow c c\right)$ & 2.7053 & 2.4096 & 3.1664 & 3.742 & 3.653 & 3.762 \\
\hline $\mathrm{BR}\left(A^{0} \rightarrow b b\right)$ & 58.646 & 52.236 & 68.611 & 80.491 & 78.769 & 80.866 \\
\hline \multicolumn{7}{|c|}{$\mathrm{BR}\left(H^{+} \rightarrow X Y\right)$ in $\%$} \\
\hline $\mathrm{BR}\left(H^{+} \rightarrow \tau^{+} \nu_{\tau}\right)$ & 5.4821 & 10.583 & 4.1585 & 3.9444 & 3.189 & 11.267 \\
\hline $\operatorname{BR}\left(H^{+} \rightarrow W^{+} h^{0}\right)$ & 89.931 & 83.481 & 87.672 & $\ldots$ & 0.058026 & $\ldots$ \\
\hline $\mathrm{BR}\left(H^{+} \rightarrow W^{+} A^{0}\right)$ & 0.025258 & $\ldots$ & 2.7961 & 93.568 & 92.76 & 83.224 \\
\hline $\mathrm{BR}\left(H^{+} \rightarrow s c\right)$ & 2.3214 & 4.5651 & 1.7396 & 1.6889 & 1.335 & 4.9918 \\
\hline $\mathrm{BR}\left(H^{+} \rightarrow b t\right)$ & 1.9984 & 0.89684 & 3.4528 & 0.623 & 2.5191 & $\ldots$ \\
\hline \multicolumn{7}{|c|}{$\operatorname{BR}(t \rightarrow X Y)$ in $\%$} \\
\hline $\mathrm{BR}\left(t \rightarrow b W^{+}\right)$ & 98.897 & 98.865 & 99.036 & 97.82 & 98.115 & 98.052 \\
\hline $\mathrm{BR}\left(t \rightarrow b H^{+}\right)$ & 0.93164 & 0.96372 & 0.79287 & 2.0086 & 1.7136 & 1.7766 \\
\hline \multicolumn{7}{|c|}{ SuperIso } \\
\hline $\operatorname{BR}\left(B \rightarrow X_{s} \gamma\right)$ & 0.00031283 & 0.00031418 & 0.00031207 & 0.00030838 & 0.00030483 & 0.00031247 \\
\hline $\mathrm{BR}\left(B_{s} \rightarrow \mu^{+} \mu^{-}\right)$ & $3.2581 \times 10^{-9}$ & $3.2422 \times 10^{-9}$ & $3.2669 \times 10^{-9}$ & $3.3121 \times 10^{-9}$ & $3.3546 \times 10^{-9}$ & $3.2634 \times 10^{-9}$ \\
\hline $\mathrm{BR}\left(B_{u} \rightarrow \tau \nu_{\tau}\right)$ & $8.1856 \times 10^{-5}$ & $8.1857 \times 10^{-5}$ & $8.1855 \times 10^{-5}$ & $8.1847 \times 10^{-5}$ & $8.1844 \times 10^{-5}$ & $8.1851 \times 10^{-5}$ \\
\hline
\end{tabular}


TABLE III. (Continued)

\begin{tabular}{|c|c|c|c|c|c|c|}
\hline Parameters & BP1 & BP2 & BP3 & BP4 & BP5 & BP6 \\
\hline \multicolumn{7}{|c|}{$\sigma \times \mathrm{BR}$ in $\mathrm{pb}$} \\
\hline$X^{t j}\left(h^{0}, \tau^{+} \tau^{-}\right)$ & 0.156455 & 0.149869 & 0.130332 & $\ldots$ & 0.000119314 & $\ldots$ \\
\hline$X^{t t}\left(h^{0}, \tau^{+} \tau^{-}\right)$ & 0.62391 & 0.597452 & 0.520465 & $\ldots$ & 0.000472038 & $\ldots$ \\
\hline$X^{t j}\left(h^{0}, b b\right)$ & 1.75101 & 1.68666 & 1.44536 & $\ldots$ & 0.00124427 & $\ldots$ \\
\hline$X^{t t}\left(h^{0}, b b\right)$ & 6.98264 & 6.72388 & 5.77187 & $\ldots$ & 0.00492267 & $\ldots$ \\
\hline$X^{t j}\left(A^{0}, \tau^{+} \tau^{-}\right)$ & $3.32498 \times 10^{-5}$ & $\ldots$ & 0.00362156 & 0.323982 & 0.274873 & 0.254534 \\
\hline$X^{t t}\left(A^{0}, \tau^{+} \tau^{-}\right)$ & 0.000132593 & $\ldots$ & 0.0144623 & 1.2779 & 1.08747 & 1.00636 \\
\hline$X^{t j}\left(A^{0}, b b\right)$ & 0.000342243 & $\ldots$ & 0.037722 & 3.75169 & 3.10504 & 2.96525 \\
\hline$X^{t t}\left(A^{0}, b b\right)$ & 0.00136479 & $\ldots$ & 0.150638 & 14.798 & 12.2843 & 11.7237 \\
\hline
\end{tabular}

discovery of charged Higgs bosons via the decay $H^{ \pm} \rightarrow$ $h^{0} l^{ \pm} \nu(l=e, \mu)$ or $H^{ \pm} \rightarrow A^{0} l^{ \pm} \nu$, via an off-shell $W^{ \pm}$ boson. To obtain a meaningful significance in our analysis, we assume the integrated luminosity of $300 \mathrm{fb}^{-1}$ and the collision energies of $\sqrt{s}=13$ and $14 \mathrm{TeV}$ at the LHC.

As mentioned, we will focus on two production channels of the charged Higgs boson; the first is $p p \rightarrow t j \rightarrow b H^{ \pm} j$ [single top (anti)quark production], and the second is $p p \rightarrow$ $t \bar{t} \rightarrow b H^{ \pm} t$ (top quark pair production). After its production, the charged Higgs boson will decay into $h^{0} W^{ \pm *}$ or $A^{0} W^{ \pm *}$, wherein the neutral Higgs state will further decay to $\tau^{+} \tau^{-}$. The $\tau^{+} \tau^{-}$decay mode can be categorized into three cases, in terms of the final states it produces, i.e., fully hadronically, fully leptonically, and semileptonically (or semihadronically), which we identify as $\tau_{\text {had }} \tau_{\text {had }}, \tau_{\text {had }} \tau_{\text {lep }}$, $\tau_{\text {lep }} \tau_{\text {lep }}$, which will be described in more detail below for each of the two production channels. Further, because in the following analysis, we do not use angular correlations of the decay products of the charged Higgs boson, our selection method can be used for both for $H^{ \pm} \rightarrow h^{0} l^{ \pm} \nu$ and $H^{ \pm} \rightarrow A^{0} l^{ \pm} \nu$ at the same time, so we will treat these two cases in the same manner. The six BPs used for the signals are provided in Table III.

Now, we present our MC analysis at a detector level, for both signal and background, which has the following features.

(i) We use MadGraph5_aMC@NLO v2.6.5 [57] to compute the cross sections and generate both signal and background events at parton level. At this level, we adopt the following kinematic cuts [in pseudorapidity, transverse momentum, and missing $E_{T}$ (MET), where $E_{T}$ is the transverse energy (or momentum)] in order to improve the efficiency of the $\mathrm{MC}$ event generation,

$$
\begin{aligned}
|\eta(l, j)|<2.5, & p_{T}(l, j)>20 \mathrm{GeV}, \\
\Delta R(l, j)>0.5, & \text { MET }>20 \mathrm{GeV},
\end{aligned}
$$

where $j$ refers here to a parton. Further, after generating the signals at the leading order (LO), we reweigh each corresponding signal event by using the next-to-LO (NLO) cross sections by $\mathrm{k}$ factors given in Table III. For the NLO cross sections of single top and top pair productions, we use $\mathrm{k}$ factors 1.06 and 1.41. In the single top production, we sum over both $p p \rightarrow t j$ and $p p \rightarrow \bar{t} j$. The $\mathrm{k}$ factors are taken from [58,59], respectively. Already by using these generation cuts, background events of pure QCD origin can be sufficiently suppressed and will be neglected in our analysis. The cross sections of signal and background processes at the LHC with $\sqrt{s}=$ $14 \mathrm{TeV}$ are presented in Table (VIII).

For the signal processes at the parton level, the full decay chains are then specified. For example, for single top production, we generate the matrix elements of the processes $p p \rightarrow t j \rightarrow H^{ \pm} b j \rightarrow h^{0}\left(A^{0}\right) W^{ \pm} b j \rightarrow$ $\tau \tau W^{ \pm} b j$ while, for top pair production, we generate the matrix elements of the processes $p p \rightarrow t \bar{t} \rightarrow$ $t H^{ \pm} b j \rightarrow t h^{0}\left(A^{0}\right) W^{ \pm} b j \rightarrow t \tau \tau W^{ \pm} b j$.

(ii) After generating both signal and background events at the parton level, we pass these events to PYTHIA v6.4 [60] to simulate initial and final state radiation (i.e., the QED and QCD emission), parton shower, hadronization, and heavy flavor decays for each of the events.

(iii) At the track (detector) level, we use DELPHES v3.4.2 [61] to simulate the detector effects and the pseudodataset generated by it to perform our analysis. To cluster final particles into jets in each event, we adopt the anti- $k_{t}$ jet algorithm [62] with jet parameter $\Delta R=0.4$ in the FastJet package [63]. The $\tau$ tagging rate is $0.7(0.6)$, and the mistagging rate for QCD jets is $0.02(0.01)$ for one-prong(multiprong) topologies [64].

From the final objects reconstructed at the detector level, i.e., $\tau$ jets, $\tau$ leptons, jets (including $b$ jets), leptons, and missing transverse momentum, we perform the signal and background discriminant analysis below. In Appendix A, in order to compare with the results of BDTG, we also provide results in a cut based method. We will consider the signal 
for the case of single (anti)top quark production and then for the case of double (anti)top quark production.

\section{A. Charged Higgs boson production from $p p \rightarrow t j$ processes}

In this subsection, we will study charged Higgs boson production and decay from single top mode. Here, charged Higgs bosons can be produced via $p p \rightarrow t j \rightarrow b H^{ \pm} j \rightarrow$ $b h^{0}\left(A^{0}\right) W^{ \pm *} j \rightarrow b h^{0}\left(A^{0}\right) l^{ \pm} \nu j \rightarrow b \tau^{+} \tau^{-} l^{ \pm} \nu j$, where $W^{ \pm *}$ denotes the off-shell $\mathrm{W}$ boson coming from the $H^{ \pm}$decaying. The BR of a top quark decaying to a charged Higgs boson can reach $1-2 \%$ or so, as shown by the BPs given in Table III. The main background processes include: 1) $p p \rightarrow W^{ \pm} \tau^{+} \tau^{-}$ production, which is generated up to two additional jets; 2) $p p \rightarrow t \bar{t}$ production, where all the top quarks decay modes are included, i.e., the two $W^{ \pm}$bosons from the top quark pair will undergo fully leptonic, semileptonic (or semihadronic), and fully hadronic decays, which are all generated up to one additional jet; 3 ) $t \bar{t} l^{+} l^{-}$production, where $l=e, \mu, \tau$; and 4) $t j \tau^{+} \tau^{-}$production.

As intimated, both signal and background events are categorized into three cases in terms of the final state emerging from the $\tau^{+} \tau^{-}$decay.

(i) Case A: both $\tau$ 's under hadronic decays. Thus, the final state includes two tagged $\tau$ jets and one lepton, $l=e, \mu$, which is from the off-shell $W^{ \pm}$decay, plus one or two extra jets.

(a) Case $\mathrm{A}$

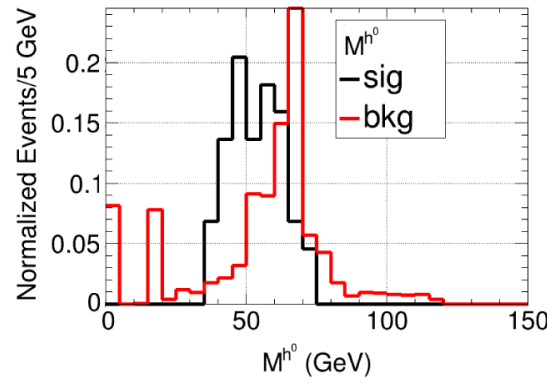

(b) Case B

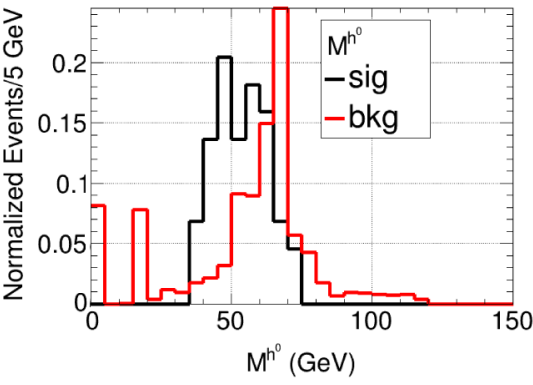

(ii) Case B: One $\tau$ undergoes hadronic decay, the other $\tau$ undergoes leptonic decay, plus there is an extra lepton from the off-shell $W^{ \pm}$decay, which is expected to be softer than the one stemming from the leptonic $\tau$ decay. Thus, the final state here is made up of two leptons, one tagged $\tau$ jet and one or two extra jets. A further requirement is that the two leptons have the same sign, which is opposite to the sign of the $\tau$ tagged jet.

(iii) Case C: Both $\tau$ 's undergo leptonic decays so that here, the final state is made up of three leptons and one (two) extra jet(s).

Below, we list some key kinematic features, which are useful to distinguish between signal and background events.

(i) The reconstructed $h^{0}$ and $A^{0}$ bosons

In a signal event, we can combine two $\tau$ jets (Case A), a $\tau$ jet, and the hardest lepton (Case B) or the two hardest leptons (Case C) to look for the mass resonance of a $h^{0}$ (for BPs 1, 2 and 3) or $A^{0}$ (for BPs 4, 5 and 6). In Case $\mathrm{A}$, the reconstructed mass should peak near the input mass of $h^{0}\left(A^{0}\right)$. In Cases $\mathrm{B}$ and $\mathrm{C}$, due to the large fraction of energy in the event taken away by neutrinos from the $\tau$ leptonic decay(s), the reconstructed mass usually peaks at a value smaller than the actual mass of the $h^{0}\left(A^{0}\right)$ state. To demonstrate this feature, in Fig. 5, we show the distribution of the invariant mass of

FIG. 5. The reconstructed neutral Higgs boson mass, $M^{h^{0}}$, is shown for Case A (a), Case B (b), and Case C (c). The signal events are generated by using BP1 in Table III.

(a) Case A

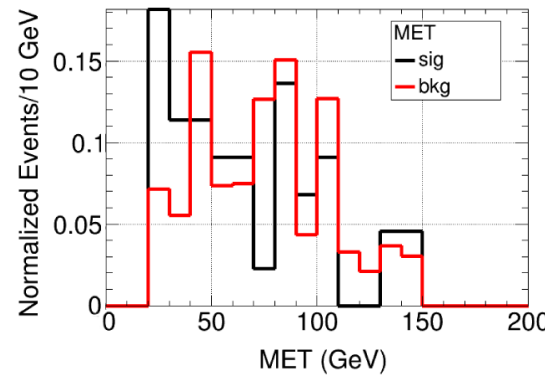

(b) Case B

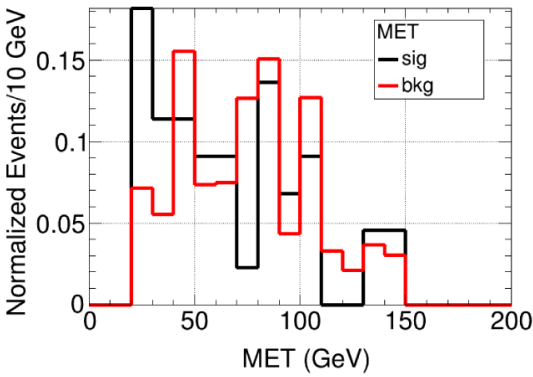

(c) Case C

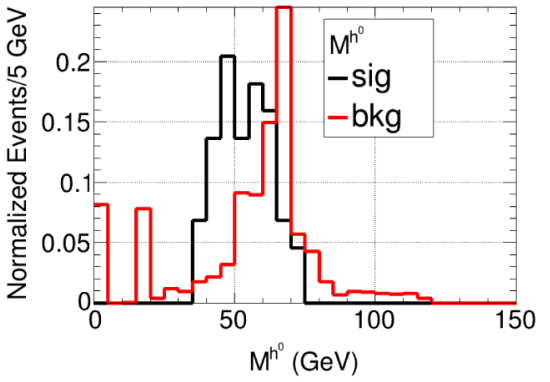

FIG. 6. The missing transverse energy, MET, is shown for Case A (a), Case B (b), and Case C (c). The signal events are generated by using BP1 in Table III. 
(a) Case A

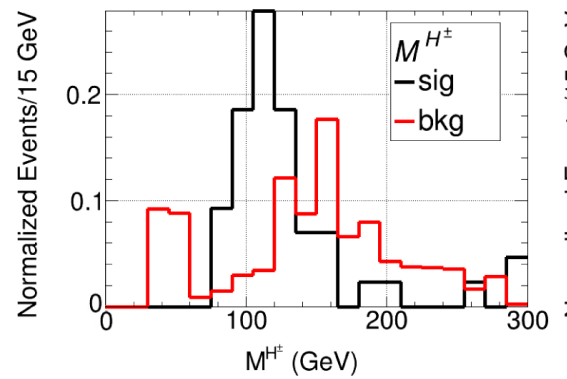

(b) Case B

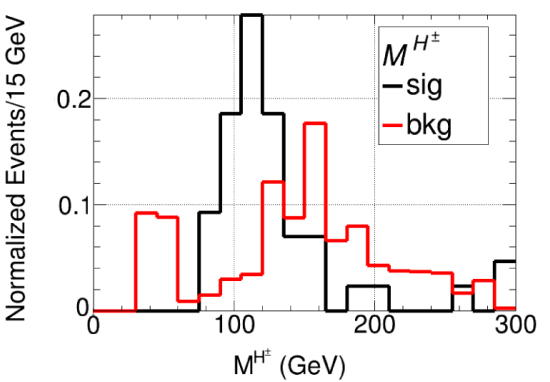

(c) Case C

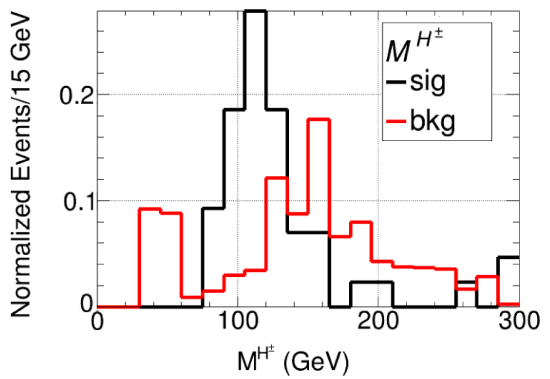

FIG. 7. The reconstructed charged Higgs boson mass, $M^{H^{ \pm}}$, is shown for Case A (a), Case B (b), and Case C (c). The signal events are generated by using BP1 in Table III.

the two $\tau$ jets for both signal and background events, denoted by $M^{h^{0}}$, where the former are generated by using BP1 in Table III, as representative of the typical signal kinematics.

(ii) The reconstructed $H^{ \pm}$boson

Since a charged Higgs boson can only transition into the final state $h^{0} l \nu$ or $A^{0} l \nu$ through an off-shell $W^{ \pm}$decay, we can attempt reconstructing its mass using the momentum of the lepton, MET, and reconstructed $h^{0}\left(A^{0}\right)$ mass. Notice that the MET of each event comes from neutrinos from $W^{ \pm}$ decays, including via leptonic $\tau$ decay. In Fig. 6 , we show its distribution for the usual three cases. In Case A, the MET mainly comes from the $W^{ \pm}$boson off-shell decay; therefore, its peak is at relatively small values, close to half of $M_{H^{ \pm}}-M_{h / A}$. In Cases $\mathrm{B}$ and $\mathrm{C}$, leptonic $\tau$ decays also contribute to the MET so that its peak value increases.

Since the $W^{ \pm}$boson coming from the $H^{ \pm}$state cannot be on shell, its decay products cannot be very energetic. Considering we have imposed an isolated and energetic requirement on the lepton, we deliberately keep the MET cut equally loose in the preselection and let a machine learning agent (see below and Appendix B) to eventually optimize it, an approach that can yield a $25 \%$ increase in the significance. The MET distribution is presented in Fig. 6. It shows that the background events have larger MET than most signal events.

We then use the MET to reconstruct the mass of the $l+\mathrm{MET}+h^{0}\left(A^{0}\right)$ system, which we denote by $M^{H^{ \pm}}$, as it is sensitive to the actual value of $M_{H^{ \pm}}$. Based on Ref. [65], we proceed as follows. We calculate the $\nu$ four momentum by enforcing the $H^{ \pm}$mass constraint on the $l \nu h^{0}\left(A^{0}\right)$ system and then, in turn, reconstruct the $H^{ \pm}$four momentum. The distribution of the ensuing charged Higgs boson mass is shown in Fig. 7. In all three cases, it is noticed that the mass peak of the charged Higgs boson can be successfully reconstructed, which can then serve as an efficient discriminant to distinguish between signal and background events.

(iii) The reconstructed $t$ quark

In a signal event, the charged Higgs boson is produced by a top quark (or antiquark) decay. Therefore, we can reconstruct the top quark mass by using the momentum of the charged Higgs boson and that of a non- $\tau$-jet. In our reconstruction method, we loop over all such jets and select the one that yields a reconstructed top quark mass, $M^{t}$, which is closest to the real one. In Fig. 8, we present its distribution.

(iv) The reconstructed $t j$ system

All non- $\tau$-jets are sorted by their transverse momentum, and then the leading two jets are considered, as mentioned above. We pick one of (a) Case $\mathrm{A}$

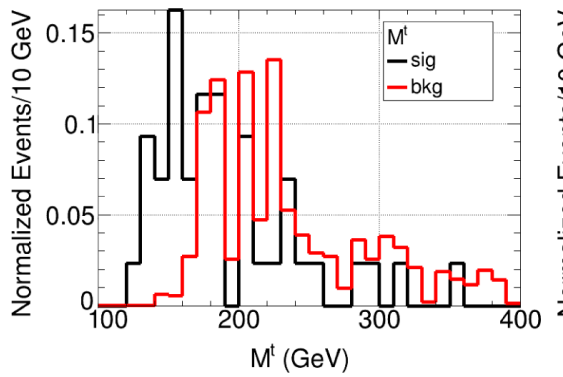

(b) Case B

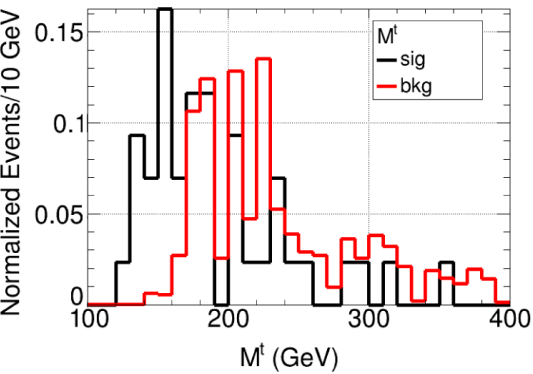

(c) Case C

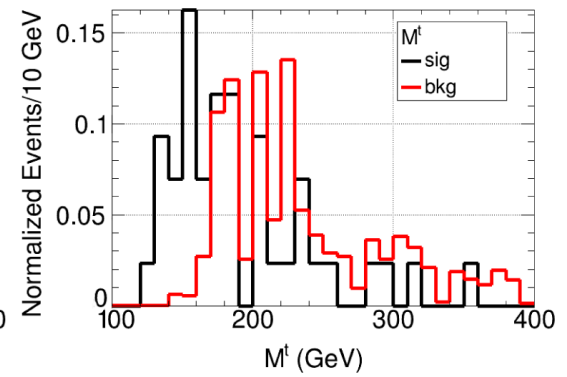

FIG. 8. The reconstructed top quark mass, $M^{t}$, is shown for Case A (a), Case B (b), and Case C (c). The signal events are generated by using BP1 in Table III. 
(a) Case $\mathrm{A}$

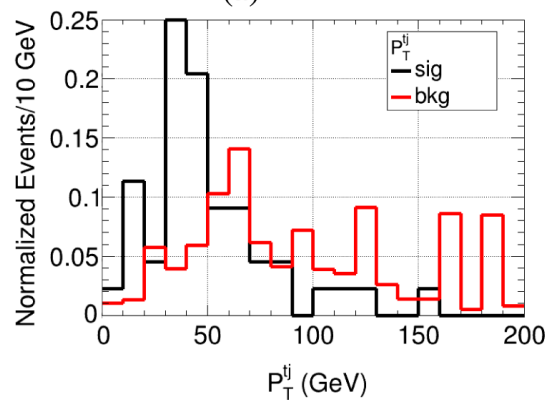

(b) Case B

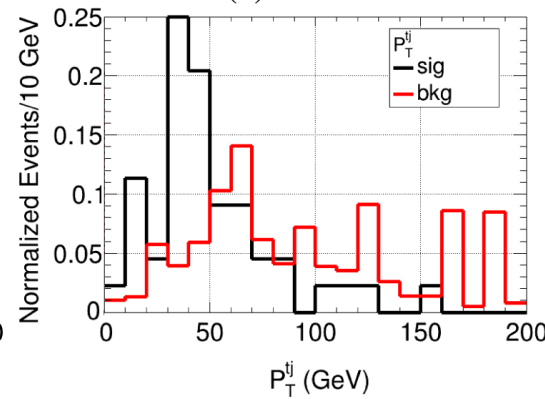

(c) Case C

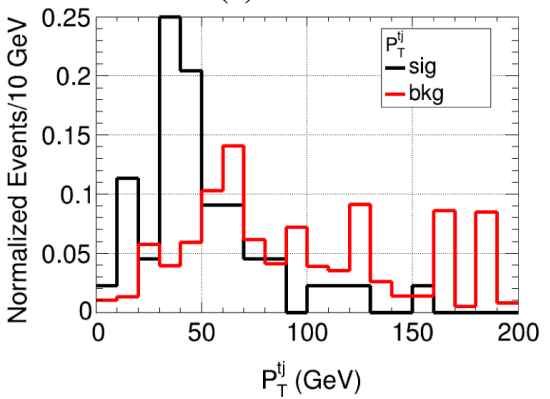

FIG. 9. The transverse momentum distributions of the reconstructed $t j, P_{T}^{t j}$, system is shown for Case A (a), Case B (b), and Case C (c). The signal events are generated by using BP1 in Table III.

(a) Case $\mathrm{A}$

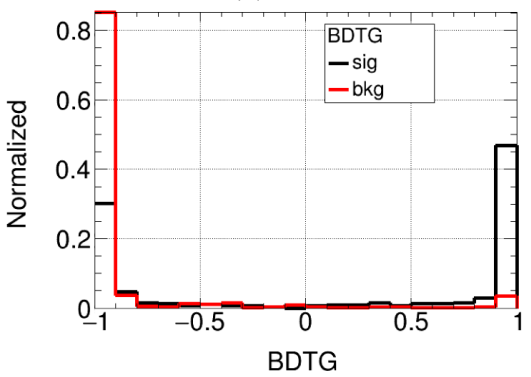

(b) Case B

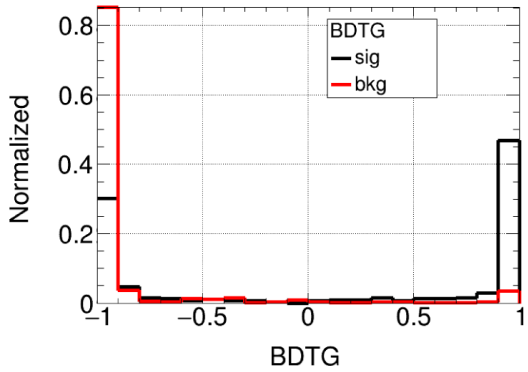

(c) Case C

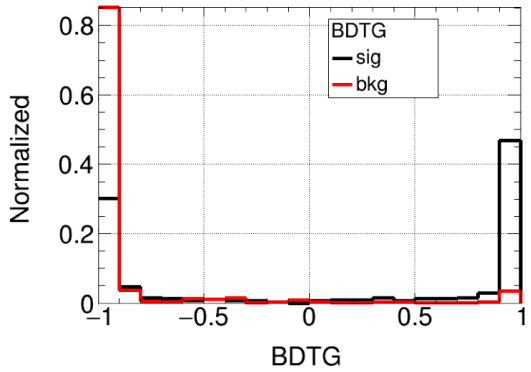

FIG. 10. The BDTG output in the $t j$ channel analysis is shown for Case A (a), Case B (b), and Case C (c). The signal events are generated by using BP1 in Table III.

the jets to reconstruct the top quark mass, then the other one is used to reconstruct the center-of-mass frame of the top-jet $(t j)$ system, which transverse momentum, $P_{T}^{t j}$, is expected to be small, as shown in Fig. 9.

To optimize the application of cuts upon the various kinematic observables we have just discussed and to improve the signal-to-background rate, as intimated, we adopt the gradient boosted decision tree (BDTG) approach, which is one of the multivariate analysis (MVA) methods. The latter has recently been widely used in data analysis and we adopt the BDTG method implemented in the toolkit for MVA (TMVA) package of ROOT [66]. In the training stage, we have used the following input variables: all final state (standard jet, $\tau$ jet, and lepton) transverse momenta, the invariant mass and transverse momentum of the $h^{0}\left(A^{0}\right)$ state, the reconstructed charged Higgs boson and top quark masses, plus the transverse momentum of the $t j$ system. The distribution of the BDTG output for both signal and background is shown in Fig. 10, from which it is noticed that the observables listed above as input are indeed efficient to distinguish between the two samples for all three Cases considered (A, B, and C). To maximally exploit the efficiency of the BDTG method, all kinematic cuts are chosen rather loose and thus, only remove the most irrelevant background events. The details of the cuts used for three cases for BP1 are summaried in Table IV, alongside the BDTG cut. We stress that the BDTG output is a much better variable to use to enhance the signal-tobackground rate than that of a traditional cutflow method based on more stringent requirements placed upon the aforementioned (or other) kinematic variables.

Although, for reasons of space, we have illustrated here only the response of BP1 (and associated background) to our selection, the pattern for the other BPs is very similar, the main difference being at the BTDG training stage where the input signal events are generated by the different kinematic parameters about each BP. Based on our selection, the final significance (which is calculated as

TABLE IV. Kinematic cuts for the analysis of BP1 in the $t j$ channel are shown.

\begin{tabular}{lccc}
\hline \hline Cuts & Case A & Case B & Case C \\
\hline 2nd jet $P_{T}$ & {$[20-130] \mathrm{GeV}$} & {$[20-150] \mathrm{GeV}$} & {$[20-200] \mathrm{GeV}$} \\
1st lepton $P_{T}$ & {$[20-60] \mathrm{GeV}$} & {$[20-60] \mathrm{GeV}$} & {$[20-100] \mathrm{GeV}$} \\
2nd lepton $P_{T}$ & $\ldots$ & {$[20-50] \mathrm{GeV}$} & {$[20-60] \mathrm{GeV}$} \\
3rd lepton $P_{T}$ & $\ldots$ & $\ldots$ & {$[20-35] \mathrm{GeV}$} \\
MET & {$[20,100] \mathrm{GeV}$} & {$[20,150] \mathrm{GeV}$} & {$[20,150] \mathrm{GeV}$} \\
$M^{h^{0}}$ & {$[40,100] \mathrm{GeV}$} & {$[10,80] \mathrm{GeV}$} & {$[20,75] \mathrm{GeV}$} \\
$M^{H^{ \pm}}$ & {$[80,300] \mathrm{GeV}$} & {$[60,250] \mathrm{GeV}$} & {$[20,160] \mathrm{GeV}$} \\
$M^{t}$ & {$[0,250] \mathrm{GeV}$} & {$[0,250] \mathrm{GeV}$} & {$[0,250] \mathrm{GeV}$} \\
BDTG & {$[0.4,1]$} & {$[-0.6,1]$} & $\ldots$ \\
\hline \hline
\end{tabular}


TABLE V. The final significances of the $t j$ channel for the six BPs considered when the luminosity is $300 \mathrm{fb}^{-1}$ at both (a) $\sqrt{s}=13 \mathrm{TeV}$ and (b) $14 \mathrm{TeV}$.

\begin{tabular}{lllllll}
\hline \hline Significance & BP1 & BP2 & BP3 & BP4 & BP5 & BP6 \\
\hline Case A & 3.33 & 2.56 & 6.01 & 6.08 & 5.47 & 4.21 \\
Case B & 2.91 & 1.85 & 4.31 & 3.86 & 3.24 & 2.91 \\
Case C & 0.73 & 0.76 & 1.40 & 0.69 & 1.13 & 0.78 \\
Combined & 3.72 & 2.94 & 7.10 & 6.77 & 6.17 & 4.83 \\
\hline \hline Significance & \multirow{2}{*}{ BP1 } & \multirow{2}{*}{ BP2 } & BP3 & BP4 & BP5 & BP6 \\
\hline Case A & 3.45 & 2.65 & 6.22 & 6.27 & 5.68 & 4.35 \\
Case B & 3.07 & 1.96 & 4.54 & 4.07 & 3.41 & 3.05 \\
Case C & 0.74 & 0.80 & 1.47 & 0.71 & 1.16 & 0.80 \\
Combined & 3.86 & 3.05 & 7.36 & 7.0 & 6.39 & 4.99 \\
\hline \hline
\end{tabular}

$S / \sqrt{S+B}$, where $S$ is the signal rate and $B$ the background one) for all Cases A, B, and C, as well as all six BPs, are summarized in Table V. ${ }^{3}$

Among these BPs, BP2 has the lowest significance because the mass difference between the charged Higgs boson $H^{ \pm}$and the light Higgs boson $h^{0}$ is the smallest, which leads to a $W^{ \pm}$boson that is far off shell (which, in turn, leads to a soft lepton). For the opposite reason, the significances in BP3, BP4, and BP5 are the highest because the mass differences between the charged and neutral Higgs bosons are very large. The event numbers in the Case $\mathrm{B}$ are very small, as can be found in details at Appendix A, because a same sign dilepton pair is required, which, however, can reduce the $t \bar{t}$ background significantly. The significances in Case $\mathrm{C}$ are mainly affected by requiring three isolated leptons and large MET when one lepton is coming from the off-shell $W^{ \pm}$boson, which is hard to detect. In Appendix B, we will prove that with softer lepton and MET cuts, there can be considerable significances also in Case C.

The combined significances for the three cases are calculated too. The luminosity is assumed to be $300 \mathrm{fb}^{-1}$ at both 13 and $14 \mathrm{TeV}$; hence, we can compare like for like the scope of Run 2 and Run 3 of the LHC, when the latter is only affected by the change of beam energy. For example, by looking at the combined rates at $14 \mathrm{TeV}$, it is clear that the significances of BP1 and BP3-6 can be larger than 2, in fact, significantly so in some cases, thus warranting a potential discovery. In contrast, BP2 can only afford one with some evidence of new physics. At $13 \mathrm{TeV}$, the results are very similar for each BPs, albeit somewhat smaller in comparison. It is noteworthy that our results are consistent with the former study presented in [67], where the main difference can be attributed to the change in the Delphes card.

\footnotetext{
${ }^{3}$ When the number of events are few, it is more appropriate to use the significance, which is defined as $\sqrt{2 \ln Q}$, where $Q=(1+S / B)^{S+B} \exp (-S)$.
}

\section{B. Charged Higgs boson production from $p p \rightarrow t t$ processes}

In this section, we extend our analysis to the $p p \rightarrow t \bar{t}$ production channel. Here, the dominant signal process can be expressed as

$$
p p \rightarrow t \bar{t} \rightarrow H^{ \pm} b \bar{b} W^{\mp} \rightarrow b \bar{b} W^{\mp} W^{ \pm *} h^{0}\left(A^{0}\right),
$$

where $W^{\mp}$ comes from the direct decaying of top quarks. While $W^{ \pm *}$ comes from $H^{ \pm}$decaying, and it can go into a pair of soft jets or a soft lepton and a soft missing energy. In our simulation, we directly consider the three-body decays of $H^{ \pm}$in the matrix element, and the decay chain is put as given below:

$$
\begin{aligned}
H^{ \pm} W^{\mp} & \rightarrow h^{0}\left(A^{0}\right) l^{ \pm} \nu W^{\mp} \rightarrow \tau^{+} \tau^{-} l^{ \pm} \nu q q, \\
\text { or } \quad H^{ \pm} W^{\mp} & \rightarrow h^{0}\left(A^{0}\right) q q W^{\mp} \rightarrow \tau^{+} \tau^{-} l^{\mp} \nu q q .
\end{aligned}
$$

For this signature, we will consider the following main background processes: 1) $t \bar{t}$ production; 2) $W^{ \pm} \tau^{+} \tau^{-}$production; 3) $t \bar{t} l^{+} l^{-}$production. (Notice that we will instead ignore the following subdominant backgrounds, which inclusive cross sections are small when compared with those of the dominant ones and, further, can be easily suppressed by our selection cuts: $W^{+} W^{-} \tau^{+} \tau^{-}$, single vector boson production processes, $W^{+} W^{-}, Z Z$, single top production, as well as multijet processes from QCD.)

Similar to the previous case, here too, events are categorized into three cases in terms of the $\tau$ decay products. In all of these cases, we employ $b$ tagging as implemented in our detector emulator.

(i) Case A: two hadronic $\tau$ decays, i.e., there are two $\tau$ jets, one soft lepton (as usual, $l=e, \mu$ ), which is from an off-shell $W^{ \pm}$decay, two $b$ jets, and two extra (untagged) jets in this signature.

(ii) Case B: one hadronic $\tau$, one leptonic $\tau$ (again, either into an electron or a muon), i.e., there are one $\tau$ jet, two leptons, two $b$ jets, and two extra (untagged) jets in the final state.

(iii) Case C: two leptonic $\tau$ decays (via the above channels), i.e., there are three leptons, two $b$ jets, and two extra (untagged) jets in this case.

Again, similarly to the analysis of the single top processes, to distinguish signal and background, we introduce the following features, which are efficient in separating the former from the latter.

(i) The reconstructed $h^{0}$ and $A^{0}$ bosons

The invariant mass of two tagged $\tau$ jets (Case A), the $\tau$ jet, and the hardest lepton (Case B) and the two hardest leptons (Case C) are clustered together. For signal events, again, the invariant mass of this system correlates to the mass of the $C P$-even or $C P$-odd Higgs boson (i.e., $h^{0}$ and $A^{0}$, respectively).

(ii) The reconstructed $W^{ \pm}$boson

The mass of the hadronic $W^{ \pm}$boson can be computed from the invariant mass of two non- $b$-jets. 
(a) Case A

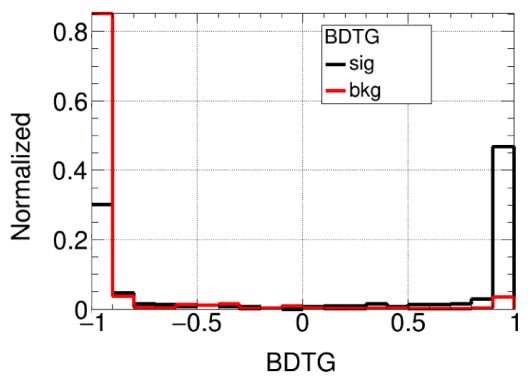

(b) Case B

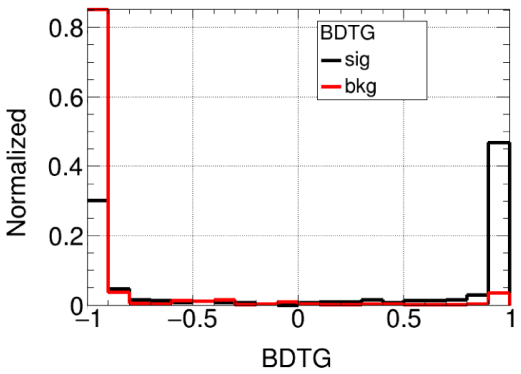

(c) Case C

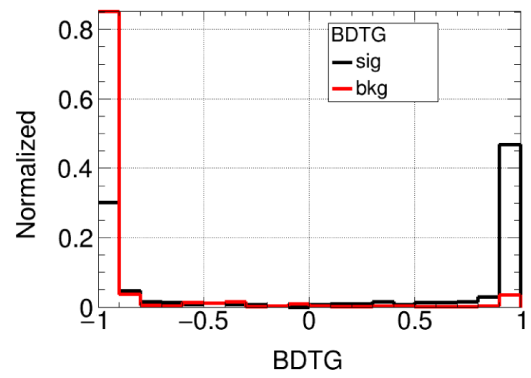

FIG. 11. The BDTG output in the $t \bar{t}$ channel analysis is shown for Case A (a), Case B (b), and Case C (c). The signal events are generated by using BP1 in Table III.

In signal events, these two non- $b$-jets are produced from hadronic $W^{ \pm}$decays and would naturally peak at $M_{W^{ \pm}}$. In the case of leptonic decays of a $W^{ \pm}$ boson, one can use instead the standard transverse mass using the softest lepton and the MET component, though for the signal, it should be recalled that the gauge boson is off shell.

(iii) The reconstructed $t$ quarks

The charged Higgs boson is not reconstructed independently, because in the signal, it could come from either $H^{ \pm} \rightarrow W^{ \pm} h^{0} \rightarrow q q \tau^{+} \tau^{-}$or $H^{ \pm} \rightarrow$ $W^{ \pm} h^{0} \rightarrow l^{ \pm} \nu \tau^{+} \tau^{-}$(similarly for the $A^{0}$ ). Instead,

TABLE VI. Kinematic cuts for the analysis of BP1 in the $t \bar{t}$ channel are shown.

\begin{tabular}{lccc}
\hline \hline Cuts & Case A & Case B & Case C \\
\hline Preselection & Preselection & Preselection & Preselection \\
\hline$M^{h^{0}}$ & {$[0,95] \mathrm{GeV}$} & {$[0,100] \mathrm{GeV}$} & {$[20,110] \mathrm{GeV}$} \\
$M^{\text {top1 }}$ & {$[100,250] \mathrm{GeV}$} & {$[90,260] \mathrm{GeV}$} & {$[70,240] \mathrm{GeV}$} \\
$M^{H^{ \pm}}$ & {$[80,220] \mathrm{GeV}$} & {$[80,200] \mathrm{GeV}$} & {$[80,200] \mathrm{GeV}$} \\
$M^{\text {top1 }}$ & {$[120,220] \mathrm{GeV}$} & {$[120,250] \mathrm{GeV}$} & {$[70,240] \mathrm{GeV}$} \\
BDTG & {$[-0.9,1]$} & {$[0,1]$} & $\ldots$ \\
\hline \hline
\end{tabular}

TABLE VII. The final significances of the $t \bar{t}$ channel for the six BPs considered when the luminosity is $300 \mathrm{fb}^{-1}$ at both (a) $\sqrt{s}=13 \mathrm{TeV}$ and (b) $14 \mathrm{TeV}$.

\begin{tabular}{llllrrr}
\hline \hline Significance & BP1 & BP2 & BP3 & BP4 & BP5 & BP6 \\
\hline Case A & 7.27 & 6.46 & 5.37 & 10.98 & 10.03 & 8.97 \\
Case B & 4.62 & 5.51 & 4.80 & 7.45 & 7.10 & 6.01 \\
Case C & 2.74 & 2.06 & 1.42 & 4.45 & 3.46 & 3.01 \\
Combined & 8.94 & 8.45 & 6.98 & 13.86 & 12.61 & 11.05 \\
\hline \hline Significance & \multirow{2}{*}{ BP1 } & \multirow{2}{*}{ BP2 } & BP3 & BP4 & BP5 & BP6 \\
\hline Case A & 7.60 & 6.74 & 5.59 & 11.45 & 10.48 & 9.35 \\
Case B & 4.85 & 5.79 & 5.04 & 7.81 & 7.47 & 6.30 \\
Case C & 2.90 & 2.15 & 1.47 & 4.62 & 3.60 & 3.12 \\
Combined & 9.35 & 8.83 & 7.27 & 14.46 & 13.18 & 11.50 \\
\hline \hline
\end{tabular}

two top quarks are reconstructed at the same time by scanning the best combination of all constituents, i.e., the combination of the reconstructed two $W^{ \pm}$ boson candidates and $h^{0} / A^{0}$ candidate given above and two $b$ jets. Contrary to the previous case of single top production, though, when only one top quark mass reconstruction is involved, here, in order to reduce the impact of combinatorics, we perform a $\chi^{2}$ fit, as follows:

$$
\chi^{2}=\left(M_{b_{i} W_{k}}-m_{t}\right)^{2}+\left(M_{b_{j} W_{l} h^{0}}-m_{t}\right)^{2},
$$

where $b_{i}, b_{j}$ loop around all $b$ jets, $W_{k}, W_{l}$ loop around two $W^{ \pm}$boson candidates, and $m_{t}$ is taken as $173 \mathrm{GeV}$. The combination which yields the minimal $\chi^{2}$ is chosen for each event.

In our BDTG method, at the training stage, we use here the following input variables: all final state ( $b$ and non$b$-jet, $\tau$ jet, and lepton) transverse momenta, the reconstructed mass of the $h^{0}\left(A^{0}\right)$ state, the reconstructed charged Higgs boson, and top quark masses. The distribution of the BDTG output is shown in Fig. 11. Again, we optimize the cuts of the BDTG output to obtain the best significance for each BP, see Table VI. The significances for each of the three cases of $\tau$ final states and the combined ones for the six BPs are presented in Table VII, for our two customary choices of energy and luminosity. At both $13 \mathrm{TeV}$ and $14 \mathrm{TeV}$, the combination of all $\tau^{+} \tau^{-}$decay modes can afford one with significant potential discovery for all BPs studied.

\section{CONCLUSIONS}

In summary, in this paper, building upon previous work of some of us, we have proven that bosonic decays of light charged Higgs bosons, i.e., $H^{ \pm} \rightarrow A^{0} W^{ \pm(*)}$ or $h^{0} W^{ \pm(*)}$, where the charged Higgs boson is produced in a top (anti) quark decay and can be accessed already during Run 2 of the LHC and certainly at Run 3. This can be done in the $\tau^{+} \tau^{-}$ decay channel of the two aforementioned neutral Higgs bosons so long that either or both of these are lighter than the SM-like Higgs boson discovered at the CERN collider back 
in 2012. This spectrum configuration is available in a $2 \mathrm{HDM}$ Type-I, wherein, it is possible to identify the latter state with the heaviest $C P$-even Higgs boson of the model, i.e., $H^{0}$. This can be achieved in the presence of all theoretical and experimental constraints presently available so that this BSM scenario is a prime candidate for a detectable new physics signal at the LHC. This requires one to exploit all possible production modes of the top (anti)quark, i.e., both single and double top (anti)quark production, and all possible $\tau^{+} \tau^{-}$ decays modes, i.e., fully hadronic, fully leptonic, and semileptonic (or semihadronic).

We have found good agreement between our results and the former study given in [67] where a dataset $100 \mathrm{fb}^{-1}$ was assumed. Considering that the Run 3 of LHC at $\sqrt{s}=$ $13 \mathrm{TeV}$ has collected a dataset around $300 \mathrm{fb}^{-1}$, we have assumed a dataset with $300 \mathrm{fb}^{-1}$ in Tables IX-XIV. These results demonstrate these 6 BPs are near the ridge to be either discovered or be ruled out.

We have shown the above to be possible by using standard cutflows, based on established triggers, acceptance. and selection requirements, eventually supplemented by a customary BDTG optimisation. Further, we have described how to reconstruct efficiently the masses of the heavy objects entering the signal processes, i.e., the $h^{0} / A^{0}$, $H^{ \pm}$and/or $t(\bar{t})$ states, depending on the production and decay channels exploited, so as to improve the signal-tobackground rates. However, the reconstruction of the $W^{ \pm}$ boson is hampered by the fact that such a state can be highly off shell so that we have also dwelt upon the possibility of improving upon the above approach by exploiting the BDTG step in order to devise a lower threshold in MET, so as to further aid the ability of extracting the signals we advocate out of the background.

We have come to the above conclusions following a complete scan of the parameter space of the 2HDM Type-I and a sophisticated MC analysis of several BPs maximizing, in turn, the $A^{0} W^{ \pm(*)}$ or $h^{0} W^{ \pm(*)}$ decay rates of the $H^{ \pm}$state. For both the former and the latter, we have used sophisticated numerical tools so that we are confident of the solidity of our results. We look forward to the ATLAS and/or CMS Collaborations to test our BSM scenarios through the advocated signatures, which may well serve the purpose of offering additional discovery modes of not only a light charged Higgs boson state but also one neutral Higgs boson state lighter than the discovered SM-like one. Unfortunately, not both $A^{0}$ and $h^{0}$ states can be accessed at the same time in this way, as the $H^{ \pm} \rightarrow A^{0} W^{ \pm(*)}$ decay rate is largest when the $H^{ \pm} \rightarrow h^{0} W^{ \pm(*)}$ one is smallest (and vice versa).

\section{ACKNOWLEDGMENTS}

Yan Wang would like to thank Felix Kling and Adarsh Pyarelal for useful communication on the Delphes card of snowmass. This work is supported by the Moroccan Ministry of Higher Education and Scientific Research MESRSFC and CNRST: Project No. PPR/2015/6. S. M. is supported also in part through the NExT Institute and the STFC consolidated Grant No. ST/L000296/1. A. A., R. B., and S.M. acknowledge the H2020-MSCA-RISE-2014 Grant No. 645722 (NonMinimalHiggs). Q. S. Yan is supported by the Natural Science Foundation of China under Grants No. 11475180 and No. 11875260. Y. W. is supported by the Scientific research funding project for introduced high level talents of the Inner Mongolia Normal University Grant No. 2019YJRC001.

\section{APPENDIX A: THE STANDARD CUTFLOW BASED ANALYSIS}

In this Appendix, for reference, we break down the standard cutflow analysis adopted in the main text in terms of its individual contributions for both signals and backgrounds. As an illustrative example, we use the case of BP1

TABLE VIII. The cross sections for signal and background processess used for the normalization of the MC event generation after the cuts $\Delta R=0.4, P_{T}^{j}>20 \mathrm{GeV}$ and $\left|\eta_{j}\right|<2.5$ at $\sqrt{s}=14 \mathrm{TeV}$.

\begin{tabular}{lccccccc}
\hline \hline Signal $t j(\mathrm{fb})$ & Signal $t \bar{t}(\mathrm{fb})$ & $W^{ \pm} \tau^{+} \tau^{-}(\mathrm{fb})$ & $t \bar{t}_{l^{+} l^{-}}(\mathrm{fb})$ & $t \bar{t}_{l \tau}(\mathrm{fb})$ & $t t_{\tau^{+} \tau^{-}}(\mathrm{fb})$ & $t t_{l q, \tau q}(\mathrm{fb})$ & $t \bar{t} l^{+} l^{-} \& t j \tau^{+} \tau^{-}(\mathrm{fb})$ \\
\hline \hline 36.5 & 244.5 & 162.2 & 15100 & 16200 & 8100 & 115080 & 21.2 \\
\hline \hline
\end{tabular}

TABLE IX. The successive efficiency in the cut based method is shown for the $t j$ channel in Case A when the luminosity is assumed to be $300 \mathrm{fb}^{-1}$ at $\sqrt{s}=14 \mathrm{TeV}$, where the digital numbers given below each process denote the numbers of events after each cut being implemented. $S$ denotes the signal events, and Total $B$ denotes the sum of all background events. The convention is same for Tables (X-XIV).

\begin{tabular}{lrrrrrrrrrr}
\hline \hline $\int L d t=300 \mathrm{fb}^{-1}$ & \multicolumn{1}{c}{$S$} & \multicolumn{1}{c}{$W^{ \pm} \tau^{+} \tau^{-}$} & \multicolumn{1}{c}{$t \bar{t}_{l^{+} l^{-}}$} & \multicolumn{1}{c}{$t \bar{t}_{l \tau}$} & \multicolumn{1}{c}{$t \bar{t}_{\tau \tau}$} & \multicolumn{1}{c}{$t \bar{t}_{l j, \tau j}$} & $t \bar{t} l^{+} l^{-} \& t j \tau^{+} \tau^{-}$ & Total $B$ & $S / B$ & $S / \sqrt{S+B}$ \\
\hline Preselection & 111.3 & 131.5 & 180.4 & 2556.7 & 282.1 & 2000.5 & 6.3 & 5157.5 & 0.02 & 1.53 \\
$M^{h^{0}} \in[40,100]$ & 102.9 & 117.8 & 65.2 & 1443.9 & 159.4 & 1059 & 4.6 & 2849.8 & 0.04 & 1.89 \\
$M^{H^{ \pm}} \in[80,300]$ & 97.5 & 97.8 & 56.7 & 1186.5 & 135.3 & 828.8 & 3.9 & 2309 & 0.04 & 1.99 \\
$M^{t} \in[0,250]$ & 85.6 & 70.1 & 28.3 & 781.7 & 92.6 & 389.6 & 2.2 & 1364.5 & 0.06 & 2.25 \\
BDTG $\in[0.4,1]$ & 59 & 9.5 & 1.9 & 98.3 & 15.9 & 110 & 0.6 & 236 & 0.25 & 3.43 \\
\hline \hline
\end{tabular}


TABLE X. The successive efficiency in the cut based method is shown for the $t j$ channel in Case B when the luminosity is assumed to be $300 \mathrm{fb}^{-1}$ at $\sqrt{s}=14 \mathrm{TeV}$.

\begin{tabular}{lcccccccccc}
\hline \hline $\int L d t=300 \mathrm{fb}^{-1}$ & $S$ & $W^{ \pm} \tau^{+} \tau^{-}$ & $t \bar{t}_{l^{+} l^{-}}$ & $t \bar{t}_{l \tau}$ & $t \bar{t}_{\tau \tau}$ & $t \bar{t}_{l j, \tau j}$ & $t \bar{t} l^{+} l^{-} \& t j \tau^{+} \tau^{-}$ & Total $B$ & $S / B$ & $S / \sqrt{S+B}$ \\
\hline Preselection & 26.4 & 51.3 & 2.8 & 22.4 & 3.8 & 0 & 3.9 & 84.3 & 0.31 & 2.51 \\
$M^{h^{0}} \in[10,80]$ & 26 & 43.8 & 1.9 & 19.2 & 3.6 & 0 & 2.9 & 71.3 & 0.36 & 2.63 \\
$M^{H^{ \pm}}(\mathrm{GeV}) \in[60,250]$ & 25 & 35.3 & 0.9 & 18.2 & 3.1 & 0 & 2.3 & 59.8 & 0.42 & 2.71 \\
$M^{t} \in[0,250]$ & 23 & 26.3 & 0.9 & 12.8 & 2.8 & 0 & 1.4 & 44.2 & 0.52 & 2.81 \\
BDTG $\in[-0.6,1]$ & 10.7 & 1.3 & 0 & 0 & 0.3 & 0 & 0.1 & 1.7 & 6.41 & 3.05 \\
\hline \hline
\end{tabular}

TABLE XI. The successive efficiency in the cut based method is shown for the $t j$ channel in Case $\mathrm{C}$ when the luminosity is assumed to be $300 \mathrm{fb}^{-1}$ at $\sqrt{s}=14 \mathrm{TeV}$.

\begin{tabular}{lcccccccccc}
\hline \hline $\int L d t=300 \mathrm{fb}^{-1}$ & $S$ & $W^{ \pm} \tau^{+} \tau^{-}$ & $t \bar{t}_{l^{+} l^{-}}$ & $t \bar{t}_{l \tau}$ & $t \bar{t}_{\tau \tau}$ & $t \bar{t}_{l j, \tau j}$ & $t \bar{t} l^{+} l^{-} \& t j \tau^{+} \tau^{-}$ & Total $B$ & $S / B$ & $S / \sqrt{S+B}$ \\
\hline Preselection & 8.6 & 15.1 & 122.7 & 25.6 & 1.5 & 79.7 & 10.6 & 255.3 & 0.03 & 0.53 \\
$M^{h^{0}} \in[20,75]$ & 8.6 & 14.2 & 100 & 22.4 & 1 & 39.9 & 8.4 & 186 & 0.05 & 0.62 \\
$M^{H^{ \pm}} \in[20,160]$ & 7.4 & 5.8 & 34.9 & 10.7 & 0.5 & 39.9 & 2.7 & 94.4 & 0.08 & 0.74 \\
$M^{t} \in[0,250]$ & 7.2 & 4.5 & 30.2 & 7.5 & 0.5 & 39.9 & 2.2 & 84.7 & 0.09 & 0.75 \\
BDTG $\in[-1,1]$ & 7.2 & 4.5 & 30.2 & 7.5 & 0.5 & 39.9 & 2.2 & 84.7 & 0.09 & 0.75 \\
\hline \hline
\end{tabular}

TABLE XII. The successive efficiency in the cut based method is tabulated for the $t \bar{t}$ channel in Case A when the luminosity is assumed to be $300 \mathrm{fb}^{-1}$ at $\sqrt{s}=14 \mathrm{TeV}$.

\begin{tabular}{lcccccccccc}
\hline \hline $\int L d t=300 \mathrm{fb}^{-1}$ & \multicolumn{1}{c}{$S$} & $W^{ \pm} \tau^{+} \tau^{-}$ & ${ }_{t} \bar{t}_{l^{+} l^{-}}$ & \multicolumn{1}{c}{$t \bar{t}_{l \tau}$} & ${ }_{t} \bar{t}_{\tau \tau}$ & $t \bar{t}_{l j, \tau j}$ & $t \bar{t} l^{+} l^{-} \& t j \tau^{+} \tau^{-}$ & Total $B$ & $S / B$ & $S / \sqrt{S+B}$ \\
\hline Preselection & 144.2 & 0.5 & 26.4 & 215.7 & 52.2 & 660.6 & 2.8 & 958.2 & 0.15 \\
$M^{A} \in[0,95]$ & 124.6 & 0.4 & 8.5 & 97.2 & 26.1 & 210.1 & 1.7 & 343.9 & 0.36 \\
$M^{t 1} \in[100,250]$ & 123.8 & 0.3 & 8.5 & 96.1 & 24.5 & 190.2 & 1.6 & 321.3 & 0.39 & 5.76 \\
$M^{H^{ \pm}} \in[80,220]$ & 109.1 & 0.2 & 3.8 & 47 & 14.8 & 60.4 & 0.8 & 127 & 0.86 & 7.1 \\
$M_{t 2} \in[120,220]$ & 87.1 & 0.02 & 0.9 & 9.6 & 3.6 & 30.2 & 0.3 & 44.7 & 1.95 \\
BDTG $\in[-0.9,1]$ & 84.7 & 0.02 & 0 & 6.4 & 2.6 & 30.2 & 0.2 & 39.4 & 2.15 \\
\hline \hline
\end{tabular}

at $\sqrt{s}=14 \mathrm{TeV}$. The cross sections for signal and background processes are given in Table VIII. The relevant listing of all data is given in Tables IX-XI for the $p p \rightarrow t j$ process and Tables XII-XII for the $p p \rightarrow t t$ channel. In these tables, $W^{ \pm} \tau^{+} \tau_{l}^{-}$is $W^{ \pm} \tau^{+} \tau^{-}$generated up to two additional jets, where the $W^{ \pm}$boson is forced to only decay into $e^{ \pm}\left(\mu^{ \pm}\right) \nu$. The $t \bar{t}$ background is separated into four groups: (i) $t \bar{t}_{l^{+} l^{-}}$: the two $W^{ \pm}$bosons in the $t \bar{t}$ channel both decay to $e^{ \pm}\left(\mu^{ \pm}\right) \nu$.

(ii) $t \bar{t}_{l \tau}$ : one $W^{ \pm}$boson decays to $e^{ \pm}\left(\mu^{ \pm}\right) \nu$ and the other $W^{ \pm}$boson decays to $\tau \nu$ whereas.

(iii) $t \bar{t}_{\tau^{+} \tau^{-}}$: the two $W^{ \pm}$bosons both decay to $\tau^{ \pm} \nu$.

(iv) $t \bar{t}_{l j, \tau j}$ : the top quark semileptonic decay, where a $W^{ \pm}$ boson decays to $e^{ \pm}\left(\mu^{ \pm}\right) \nu$ or $\tau^{ \pm} \nu$ and the other $W^{\mp}$ boson decays to quarks.

TABLE XIII. The successive efficiency in the cut based method is tabulated for the $t \bar{t}$ channel in Case B when the luminosity is assumed to be $300 \mathrm{fb}^{-1}$ at $\sqrt{s}=14 \mathrm{TeV}$.

\begin{tabular}{lcccccccccc}
\hline \hline $\int L d t=300 \mathrm{fb}^{-1}$ & $S$ & $W^{ \pm} \tau^{+} \tau^{-}$ & $t_{l_{l^{+}} l^{-}}$ & $t \bar{t}_{l \tau}$ & $\bar{t}_{\tau \tau}$ & $t \bar{t}_{l j, \tau j}$ & $t \bar{t} l^{+} l^{-} \& t j \tau^{+} \tau^{-}$ & Total $B$ & $S / B$ & $S / \sqrt{S+B}$ \\
\hline Preselection & 45.6 & 0.4 & 0 & 2.1 & 1.5 & 0 & 2.4 & 6.5 & 7.04 \\
$M^{A} \in[13,100]$ & 40.7 & 0.3 & 0 & 1.1 & 1 & 0 & 1.5 & 3.9 & 10.35 \\
$M^{t 1} \in[90,260]$ & 39.9 & 0.2 & 0 & 1.1 & 1 & 0 & 1.3 & 3.7 & 10.92 \\
$M^{H^{ \pm}} \in[80,200]$ & 36.7 & 0.2 & 0 & 1.1 & 1 & 0 & 0.5 & 2.8 & 13.12 & 5.84 \\
$M_{t 2} \in[120,250]$ & 33.4 & 0.04 & 0 & 1.1 & 1 & 0 & 0.3 & 2.4 & 13.71 \\
BDTG $\in[0,1]$ & 23.6 & 0 & 0 & 0 & 0 & 0 & 0.08 & 0.08 & 309.65 \\
\hline \hline
\end{tabular}


TABLE XIV. The successive efficiency in the cut based method is tabulated for the $t \bar{t}$ channel in Case $\mathrm{C}$ when the luminosity is assumed to be $300 \mathrm{fb}^{-1}$ at $\sqrt{s}=14 \mathrm{TeV}$.

\begin{tabular}{lcccccccccc}
\hline \hline $\int L d t=300 \mathrm{fb}^{-1}$ & \multicolumn{1}{c}{$S$} & $W^{ \pm} \tau^{+} \tau^{-}$ & $t \bar{t}_{l^{+} l^{-}}$ & $t \bar{t}_{l \tau}$ & $t \bar{t}_{\tau \tau}$ & $t \bar{t}_{l j, \tau j}$ & $t \bar{t} l^{+} l^{-} \& t j \tau^{+} \tau^{-}$ & Total $B$ & $S / B$ & $S / \sqrt{S+B}$ \\
\hline Preselection & 22.8 & 0.04 & 19.8 & 5.3 & 0.5 & 39.8 & 6.4 & 72 & 0.32 & 2.34 \\
$M^{A} \in[20,110]$ & 22.8 & 0.04 & 18.9 & 3.2 & 0.5 & 0 & 6.2 & 28.9 & 0.79 & 3.17 \\
$M^{t 1} \in[70,240]$ & 22.8 & 0.04 & 17 & 3.2 & 0.5 & 0 & 5.7 & 26.5 & 0.86 & 3.25 \\
$M^{H^{ \pm} \in[80,200]}$ & 18.7 & 0.02 & 12.3 & 2.1 & 0.5 & 0 & 4 & 18.9 & 0.99 & 3.05 \\
$M_{t 2} \in[70,240]$ & 13 & 0 & 3.8 & 1.1 & 0.5 & 0 & 1.8 & 7.2 & 1.82 & 2.9 \\
BDTG $\in[-1,1]$ & 13 & 0 & 3.8 & 1.1 & 0.5 & 0 & 1.8 & 7.2 & 01.82 & 2.9 \\
\hline \hline
\end{tabular}

Notice that $t \bar{t}$ decays to quark only channels (the fullhadronic decay mode) are very small after the preselection already and totally negligible after the cuts around the resonances; thus, they are not shown. The cuts are applied to increase the significance $(S / \sqrt{S+B})$ for the most of the cases. But for some special cases, i.e., Case B and Case C in $p p \rightarrow t t$ process, due to the fact that the event number is not enough, the BDTG can't be trained very well. Thus, the cuts are applied to increase the variable $S / B$. The BDTG cut and the $M_{t 2}$ cut are still applied for these two cases because when the simulated event number increase, these cuts would become valuable.

\section{APPENDIX B: A LOOSER PRESELECTION BASED ANALYSIS}

As mentioned in the main text, several useful observables are related to the successful reconstruction of the offshell $W^{ \pm}$bosons. Typically, each lepton from the off-shell $W^{ \pm}$bosons takes off almost half of the available energy, i.e., $M_{H^{ \pm}}-M_{h^{0}\left(A^{0}\right)}$, which can vary from 30 to $60 \mathrm{GeV}$ in the BPs given in Table III. Therefore, when a tight cut is applied, as done in the standard cutflow illustrated in the previous Appendix, signal events can suffer a bigger reduction than background ones. For example, when the MET cut is changed to be $10 \mathrm{GeV}$ (down from $20 \mathrm{GeV}$ ), we notice that the significance can already be improved by $20 \%$ or so.

Hence, here, we recompute the significance for each BP by using softer preselection cuts in general, i.e., $P_{T}(l)>$ $10 \mathrm{GeV}$ and MET $>5 \mathrm{GeV}$ (while maintaining the original pseudorapidity restriction), with the BDTG having optimized these. (Again, we only present results for the $\sqrt{s}=14 \mathrm{TeV}$ case.) The corresponding significances for single (anti)top production are given in Table XV, while
TABLE XV. The final significances of the $t j$ channel for the six BPs considered when the luminosity is $300 \mathrm{fb}^{-1}$ at $14 \mathrm{TeV}$ with a looser preselection cut are demonstrated.

\begin{tabular}{lrrrrrr}
\hline \hline Significance & \multicolumn{1}{c}{ BP1 } & BP2 & BP3 & BP4 & BP5 & BP6 \\
\hline Case A & 7.43 & 4.49 & 11.15 & 10.72 & 11.10 & 8.80 \\
Case B & 6.86 & 3.36 & 6.04 & 10.51 & 10.84 & 9.58 \\
Case C & 4.85 & 3.69 & 6.88 & 8.00 & 8.24 & 7.43 \\
Combined & 10.91 & 6.33 & 14.34 & 16.37 & 16.96 & 14.24 \\
\hline \hline
\end{tabular}

TABLE XVI. The final significances of the $t \bar{t}$ channel for the six BPs considered when the luminosity is $300 \mathrm{fb}^{-1}$ at $14 \mathrm{TeV}$ with a looser preselection cut are demonstrated.

\begin{tabular}{lcccrrr}
\hline \hline Significance & BP1 & BP2 & BP3 & BP4 & BP5 & BP6 \\
\hline Case A & 11.05 & 11.47 & 9.66 & 18.0 & 15.88 & 16.32 \\
Case B & 10.27 & 11.33 & 9.787 & 14.98 & 12.92 & 10.18 \\
Case C & 6.409 & 7.31 & 5.30 & 8.16 & 8.69 & 9.42 \\
Combine & 16.32 & 17.63 & 14.41 & 23.78 & 22.08 & 21.03 \\
\hline \hline
\end{tabular}

results for (anti)top quark pair production are given in Table XVI. Compared with the values given in Table V and VII in the main text, respectively, the significances shown here display an improvement by $30 \%$ or so.

Nevertheless, such an improvement might not be taken as granted, since we have not taken into account the possible additional background contributions from QCD processes, like heavy flavor quark production and decay, which can mimic the signal when the cuts on $P_{T}(l)$ and MET are thus loosened. Since we cannot generate the required number of background events, a more realistic analysis should be performed, and we advocate this to be attempted by the LHC experimental groups. 
[1] G. Aad et al. (ATLAS Collaboration), Phys. Lett. B 716, 1 (2012).

[2] S. Chatrchyan et al. (CMS Collaboration), Phys. Lett. B 716, 30 (2012).

[3] G. Aad et al. (ATLAS and CMS Collaborations), J. High Energy Phys. 08 (2016) 045.

[4] V. D. Barger, R. J. N. Phillips, and D. P. Roy, Phys. Lett. B 324, 236 (1994); J. F. Gunion, H. E. Haber, F. E. Paige, W. K. Tung, and S. S. D. Willenbrock, Nucl. Phys. B294, 621 (1987); R. M. Barnett, H. E. Haber, and D. E. Soper, Nucl. Phys. B306, 697 (1988); J. L. Diaz-Cruz and O. A. Sampayo, Phys. Rev. D 50, 6820 (1994); D. J. Miller, S. Moretti, D. P. Roy, and W. J. Stirling, Phys. Rev. D 61, 055011 (2000); S. Moretti and D. P. Roy, Phys. Lett. B 470, 209 (1999); E. L. Berger, T. Han, J. Jiang, and T. Plehn, Phys. Rev. D 71, 115012 (2005); M. Flechl, R. Klees, M. Kramer, M. Spira, and M. Ubiali, Phys. Rev. D 91, 075015 (2015); C. Degrande, M. Ubiali, M. Wiesemann, and M. Zaro, J. High Energy Phys. 10 (2015) 145; C. Degrande, R. Frederix, V. Hirschi, M. Ubiali, M. Wiesemann, and M. Zaro, Phys. Lett. B 772, 87 (2017).

[5] D. A. Dicus, J. L. Hewett, C. Kao, and T. G. Rizzo, Phys. Rev. D 40, 787 (1989); A. A. B. Bendezu and B. A. Kniehl, Phys. Rev. D 59, 015009 (1998); A. A. B. Bendezu and B. A. Kniehl, Phys. Rev. D 63, 015009 (2000); W. Hollik and S. h. Zhu, Phys. Rev. D 65, 075015 (2002); O. Brein, W. Hollik, and S. Kanemura, Phys. Rev. D 63, 095001 (2001).

[6] Q. H. Cao, S. Kanemura, and C. P. Yuan, Phys. Rev. D 69, 075008 (2004).

[7] A. C. Bawa, C. S. Kim, and A. D. Martin, Z. Phys. C 47, 75 (1990); A. A. B. Bendezu and B. A. Kniehl, Nucl. Phys. B568, 305 (2000); A. Krause, T. Plehn, M. Spira, and P. M. Zerwas, Nucl. Phys. B519, 85 (1998).

[8] S. Moretti and K. Odagiri, Phys. Rev. D 55, 5627 (1997); A. Arhrib, K. Cheung, J. S. Lee, and C. T. Lu, J. High Energy Phys. 05 (2016) 093.

[9] S. Dittmaier, G. Hiller, T. Plehn, and M. Spannowsky, Phys. Rev. D 77, 115001 (2008); J. Hernandez-Sanchez, S. Moretti, R. Noriega-Papaqui, and A. Rosado, J. High Energy Phys. 07 (2013) 044.

[10] M. Aoki, R. Guedes, S. Kanemura, S. Moretti, R. Santos, and K. Yagyu, Phys. Rev. D 84, 055028 (2011).

[11] A. G. Akeroyd et al., Eur. Phys. J. C 77, 276 (2017).

[12] A. M. Sirunyan et al. (CMS Collaboration), J. High Energy Phys. 07 (2019) 142; V. Khachatryan et al. (CMS Collaboration), J. High Energy Phys. 11 (2015) 018; CMS Collaboration, Search for charged Higgs bosons with the $\mathrm{H}^{ \pm} \rightarrow \tau^{ \pm} \nu_{\tau}$ decay channel in the fully hadronic final state at $\sqrt{s}=13 \mathrm{TeV}$, Report No. CMS-PAS-HIG-16-031; CMS Collaboration, Report No. CMS-PAS-HIG-18-014.

[13] G. Aad et al. (ATLAS Collaboration), J. High Energy Phys. 03 (2015) 088; M. Aaboud et al. (ATLAS Collaboration), J. High Energy Phys. 09 (2018) 139; M. Aaboud et al. (ATLAS Collaboration), Phys. Lett. B 759, 555 (2016); G. Aad et al. (ATLAS Collaboration), J. High Energy Phys. 03 (2015) 088.

[14] G. Aad et al. (ATLAS Collaboration), Eur. Phys. J. C 73, 2465 (2013); M. Flechl (ATLAS Collaboration), Proc. Sci., EPS-HEP2011 (2011) 243 [arXiv:1307.2045].
[15] A. M. Sirunyan et al. (CMS Collaboration), Phys. Rev. D 102, 072001 (2020); A. M. Sirunyan et al. (CMS Collaboration), J. High Energy Phys. 11 (2018) 115; V. Khachatryan et al. (CMS Collaboration), J. High Energy Phys. 12 (2015) 178.

[16] A. G. Akeroyd, S. Moretti, and M. Song, Phys. Rev. D 101, 035021 (2020).

[17] A. Arhrib, R. Benbrik, and S. Moretti, Eur. Phys. J. C 77, 621 (2017).

[18] S. L. Glashow and S. Weinberg, Phys. Rev. D 15, 1958 (1977).

[19] G. C. Branco, P. M. Ferreira, L. Lavoura, M. N. Rebelo, M. Sher, and J. P. Silva, Phys. Rep. 516, 1 (2012).

[20] J. F. Gunion, H. E. Haber, G. L. Kane, and S. Dawson, Front. Phys. 80, 1 (2000).

[21] N. G. Deshpande and E. Ma, Phys. Rev. D 18, 2574 (1978).

[22] A. G. Akeroyd, A. Arhrib, and E. M. Naimi, Phys. Lett. B 490, 119 (2000); S. Kanemura, T. Kubota, and E. Takasugi, Phys. Lett. B 313, 155 (1993); P. M. Ferreira, R. Santos, and A. Barroso, Phys. Lett. B 603, 219 (2004); 629, 114 (2005).

[23] K. Olive et al., Chin. Phys. C 38, 090001 (2014).

[24] D. Eriksson, J. Rathsman, and O. Stal, Comput. Phys. Commun. 181, 189 (2010).

[25] F. Mahmoudi, Comput. Phys. Commun. 180, 1579 (2009).

[26] Y. Amhis et al., arXiv:1412.7515.

[27] Heavy Flavor Averaging Group, www.slac.stanford.edu/ xorg/hfag/rare/2013/radll/btosg.pdf.

[28] Heavy Flavor Averaging Group, www.slac.stanford.edu/ xorg/hfag/rare/2014/bs/index.html.

[29] Heavy Flavor Averaging Group, www.slac.stanford.edu/ xorg/hfag/rare/2014/radll/OUTPUT/HTML/radll_table4 .html.

[30] M. Tanabashi et al. (Particle Data Group), Phys. Rev. D 98, 030001 (2018).

[31] P. Bechtle, S. Heinemeyer, O. Stal, T. Stefaniak, and G. Weiglein, Eur. Phys. J. C 74, 2711 (2014).

[32] O. Stål and T. Stefaniak, Proc. Sci., EPS-HEP2013 (2013) 314 [arXiv:1310.4039].

[33] (ATLAS Collaboration), Report No. ATLAS-CONF-2016112.

[34] (ATLAS Collaboration), Report No. ATLAS-CONF-2018004.

[35] M. Aaboud et al. (ATLAS Collaboration), J. High Energy Phys. 12 (2017) 024.

[36] M. Aaboud et al. (ATLAS Collaboration), J. High Energy Phys. 03 (2018) 095.

[37] M. Aaboud et al. (ATLAS Collaboration), Phys. Rev. D 97, 072003 (2018).

[38] M. Aaboud et al. (ATLAS Collaboration), Phys. Rev. D 97, 072016 (2018).

[39] M. Aaboud et al. (ATLAS Collaboration), Phys. Rev. D 98, 052005 (2018).

[40] CMS Collaboration, Report No. CMS-PAS-HIG-16-040.

[41] A. M. Sirunyan et al. (CMS Collaboration), J. High Energy Phys. 11 (2017) 047.

[42] A. M. Sirunyan et al. (CMS Collaboration), Phys. Lett. B 779, 283 (2018).

[43] A. M. Sirunyan et al. (CMS Collaboration), Phys. Rev. Lett. 120, 071802 (2018). 
[44] A. M. Sirunyan et al. (CMS Collaboration), Phys. Lett. B 780, 501 (2018).

[45] A. M. Sirunyan et al. (CMS Collaboration), J. High Energy Phys. 08 (2018) 066.

[46] A. M. Sirunyan et al. (CMS Collaboration), J. High Energy Phys. 06 (2018) 101.

[47] A. M. Sirunyan et al. (CMS Collaboration), J. High Energy Phys. 03 (2019) 026.

[48] A. M. Sirunyan et al. (CMS Collaboration), Phys. Lett. B 791, 96 (2019).

[49] A. M. Sirunyan et al. (CMS Collaboration), Phys. Rev. Lett. 122, 021801 (2019).

[50] P. Bechtle, O. Brein, S. Heinemeyer, O. Stal, T. Stefaniak, G. Weiglein, and K. E. Williams, Eur. Phys. J. C 74, 2693 (2014).

[51] A. Arhrib, R. Benbrik, S. Moretti, A. Rouchad, Q. S. Yan, and X. Zhang, J. High Energy Phys. 07 (2018) 007.

[52] S. Moretti, R. Santos, and P. Sharma, Phys. Lett. B 760, 697 (2016).

[53] S. Moretti, R. Santos, and P. Sharma, Proc. Sci., CHARGED2016 (2016) 025 [arXiv:1611.09082].

[54] A. Arhrib, R. Benbrik, S. Moretti, R. Santos, and P. Sharma, Phys. Rev. D 97, 075037 (2018).

[55] A. Arhrib, R. Benbrik, R. Enberg, W. Klemm, S. Moretti, and S. Munir, Phys. Lett. B 774, 591 (2017).
[56] M. Aaboud et al. (ATLAS Collaboration), J. High Energy Phys. 09 (2018) 139.

[57] J. Alwall, R. Frederix, S. Frixione, V. Hirschi, F. Maltoni, O. Mattelaer, H.-S. Shao, T. Stelzer, P. Torrielli, and M. Zaro, J. High Energy Phys. 07 (2014) 079.

[58] B. W. Harris, E. Laenen, L. Phaf, Z. Sullivan, and S. Weinzierl, Phys. Rev. D 66, 054024 (2002).

[59] K. Melnikov and M. Schulze, J. High Energy Phys. 08 (2009) 049.

[60] T. Sjostrand, S. Mrenna, and P. Z. Skands, J. High Energy Phys. 05 (2006) 026.

[61] J. de Favereau, C. Delaere, P. Demin, A. Giammanco, V. Lemaître, A. Mertens, and M. Selvaggi (DELPHES 3 Collaboration), J. High Energy Phys. 02 (2014) 057.

[62] M. Cacciari, G. P. Salam, and G. Soyez, J. High Energy Phys. 04 (2008) 063.

[63] M. Cacciari, G. P. Salam, and G. Soyez, Eur. Phys. J. C 72, 1896 (2012).

[64] The ATLAS Collaboration, Report No. ATL-PHYS-PUB2015-045.

[65] G. Aad et al. (ATLAS Collaboration), Phys. Lett. B 717, 330 (2012).

[66] I. Antcheva et al., Comput. Phys. Commun. 182, 1384 (2011).

[67] F. Kling, A. Pyarelal, and S. Su, J. High Energy Phys. 11 (2015) 051. 